\title{
Controlling the object phase for g-factor reduction in phase-constrained parallel MRI using spatially selective RF pulses
}

Adam O. Kettinger ${ }^{1,2 *}$, Stephan A. R. Kannengiesser ${ }^{3}$, Felix A. Breuer ${ }^{4}$, Zoltan Vidnyanszky ${ }^{2}$, Martin Blaimer $^{4}$

${ }^{1}$ Department of Nuclear Techniques, Budapest University of Technology and Economics, Budapest, Hungary

${ }^{2}$ Brain Imaging Centre, Research Centre for Natural Sciences, Hungarian Academy of Sciences, Budapest, Hungary

${ }^{3}$ Siemens Healthcare GmbH, Erlangen, Germany

${ }^{4}$ Magnetic Resonance and X-ray Imaging Department, Fraunhofer Development Center X-ray Technology (EZRT), Würzburg, Germany

*Corresponding author: Adam Otto Kettinger, Department of Nuclear Techniques, Budapest University of Technology and Economics, 1111 Budapest, tel. +36307543511, kettinger.adam@gmail.com

\section{Submitted to Magnetic Resonance in Medicine}

Published version: http://onlinelibrary.wiley.com/doi/10.1002/mrm.26890/abstract 


\begin{abstract}
$\underline{\text { Purpose }}$

Parallel imaging generally entails a reduction in the signal-to-noise ratio (SNR) of the final image. Phaseconstrained methods aim to improve reconstruction quality by employing symmetry properties of k-space. Noise amplification in phase-constrained reconstruction heavily depends on the object background phase. The purpose of this work is to present a new approach of using tailored RF pulses to optimize the object phase distribution in order to maximize the benefit of phase-constrained reconstruction, and minimize the noise amplification.

Methods

Intrinsic object phase and coil sensitivity profiles are measured in a prescan. Optimal phase distribution is computed to maximize SNR in the given setup. Tailored RF pulses are designed to introduce the optimal phase map in the following accelerated acquisitions, subsequently reconstructed by phase-constrained methods. The potential of the method is demonstrated in vivo with in-plane accelerated (8x) and simultaneous multi-slice (3x) acquisitions.

Results

Mean g-factors are reduced by up to a factor of 2 compared to conventional techniques when an appropriate phase-constrained reconstruction is applied to phase-optimized acquisitions, enhancing the SNR of the final images and the visibility of small details.

Conclusion
\end{abstract}

Combining phase-constrained reconstruction and phase optimization by tailored RF pulses can provide notable improvement in the SNR and reconstruction quality of accelerated MRI.

\title{
Keywords
}

Parallel MRI, phase-constrained, GRAPPA, Multiband, RF pulses 


\section{Introduction}

Parallel imaging (PI) methods are widely used in MRI to reduce scan time by undersampling k-space and using the spatial information of receiver arrays to recover the missing information. Traditional Cartesian PI is typically performed by omitting some phase-encoding (PE) lines during the acquisition, using dedicated reconstruction techniques like SENSE (1) or GRAPPA (2) to remove the arising aliasing artifacts. However, scan time reduction with PI comes at a cost of decreased signal-to-noise ratio (SNR) of the final image, formed by an inherent loss due to the reduced amount of measured data, and a spatially varying noise amplification caused by the reconstruction. The latter is usually characterized by the g-factor $(1,3)$, and depends on the measurement setup, the scanned object, the acceleration pattern, the coil array geometry, and the reconstruction algorithm.

Simultaneous multi-slice (SMS) imaging (4-7) uses receiver arrays to disentangle simultaneously measured slices. The larger number of excited spins increases the measurement signal, or from another point of view, the higher number of phase-encoding lines per voxel reduces the image noise, eliminating the intrinsic SNR loss, causing the SMS methods to evoke strong interest recently. Nevertheless, the noise amplification caused by the reconstruction is present with any kind of PI used, and often is the main limitation on the feasible acceleration factor. For this reason, several methods were proposed to reduce the g-factors and thus increase the SNR.

One group of such methods is the so-called phase-constrained imaging (8-14). These techniques use symmetry properties of $\mathrm{k}$-space or a-priori phase information to recover missing information. A wellknown example is partial Fourier imaging (12-14) where one half of k-space is only partially acquired, and the missing data are calculated by enforcing complex conjugate symmetry. Other phase-constrained methods improve the conditioning of PI by incorporating a-priori phase information into the reconstruction $(9,11)$.

Recently, an additional phase-constrained technique called virtual conjugate coil (VCC) concept (10), was proposed. It exploits conjugate symmetry to improve reconstruction quality by creating virtual coil signals that can be easily inserted into conventional PI algorithms. Also, when combined with GRAPPA, the VCC method does not require explicit phase information (10), preserves the object phase (15), and is able to reduce the noise amplification in both traditional PI (10) and SMS imaging (16). Since VCC is a phase-constrained technique, the associated g-factors are highly dependent on the object background phase. It has been shown by simulation (10) that an optimal object phase map exists that minimizes the g-factors in the given measurement setup. Simple experiments approximated the optimal phase by a spatially linear phase-ramp achieved by a phase-encoding gradient offset (10). Initial experiments using 
spatially selective pulses to introduce the optimal object phase for in-plane acceleration have been presented in (17) using a preclinical device.

In this work, we approximate the optimal phase distribution using tailored RF pulses to reduce the noise amplification in both in-plane PI and SMS acquisitions, on a clinical scanner. The theoretical background of the method is described, and in-vivo measurements are presented. The resulting image quality is compared with conventional PI reconstructions and phase-constrained reconstruction with 1D linear phase control.

\section{Theory}

\section{$\underline{\text { Review: Virtual conjugate coil concept }}$}

In the following, a review of the VCC technique $(10,15)$ is given, as it is the theoretical background of this paper.

Denoting the spatial position by $\mathbf{r}$, the signal from the $j$-th coil element with sensitivity profile $C_{j}(\mathbf{r})$ is the Fourier transform of the spin density - divided into $\rho(\mathbf{r})$ magnitude and $e^{i \varphi(\mathbf{r})}$ phase -, weighted by the sensitivity. The idea of VCC is to incorporate the object background phase into the sensitivity profile, thus creating an effective sensitivity $D_{j}(\mathbf{r})(8,10)$. This way, the signal can be thought of as the Fourier transform of the magnitude of the spin density multiplied with the effective sensitivity:

$$
S_{j}(\mathbf{k})=\int \rho(\mathbf{r}) e^{i \varphi(\mathbf{r})} C_{j}(\mathbf{r}) e^{-i 2 \pi \mathbf{k r}} d \mathbf{r}=\mathcal{F}\left\{\rho(\mathbf{r}) e^{i \varphi(\mathbf{r})} C_{j}(\mathbf{r})\right\}=\mathcal{F}\left\{\rho(\mathbf{r}) D_{j}(\mathbf{r})\right\}
$$

The complex conjugate of the signal from the symmetric counterpart k-space location is given by:

$$
S_{j}^{*}(-\mathbf{k})=\int \rho(\mathbf{r}) e^{-i \varphi(\mathbf{r})} C_{j}^{*}(\mathbf{r}) e^{i 2 \pi(-\mathbf{k}) \mathbf{r}} d \mathbf{r}=\mathcal{F}\left\{\rho(\mathbf{r}) D_{j}^{*}(\mathbf{r})\right\}
$$

This signal can be thought of as a signal measured in the original location $\mathbf{k}$ using a physically nonexistent, virtual coil with effective sensitivity profile $D_{j}^{*}$. If the effective sensitivity is purely real everywhere in the image-domain, then this is equal to the original signal. However, if the effective sensitivity has an imaginary component, which is usually the case in normal imaging situations, then this new signal can provide additional information and thus can improve the conditioning of the reconstruction. The extent of benefit strongly depends on the phase of the effective sensitivity, i.e. the phase of the physical sensitivities, and more importantly, the object phase. The influence of these phase profiles on the reconstruction is explicitly described later.

Incorporating the VCC signals into the reconstruction 
The integration of the VCC signals into standard PI reconstruction algorithms like SENSE and GRAPPA is summarized here for clarity. The following applies for both in-plane and 3D PI as well as SMS measurements, using either SENSE (1), GRAPPA (2), SENSE/GRAPPA hybrid (18), Slice-GRAPPA (6), SPIRiT (19), or ESPIRiT (20) reconstruction methods.

\section{VCC-SENSE}

VCC-SENSE can be derived from the original SENSE equation by incorporating the object phase into the sensitivity profiles. For a given overlapping voxel set, with the image-space coil signals $\mathbf{s}$, the sensitivity matrix $\mathbf{C}$, the effective sensitivity matrix $\mathbf{D}$, the complex-valued spin density $\widetilde{\boldsymbol{\rho}}$, and the magnitude of the spin density $\boldsymbol{\rho}$, using $N$ coils and acceleration factor $R$ :

$$
\mathbf{D}=\left[\begin{array}{ccc}
C_{1,1} e^{i \varphi_{1}} & \cdots & C_{1, R} e^{i \varphi_{R}} \\
\vdots & \ddots & \vdots \\
C_{N, 1} e^{i \varphi_{1}} & \cdots & C_{N, R} e^{i \varphi_{R}}
\end{array}\right], \quad \widetilde{\boldsymbol{\rho}}=\left[\begin{array}{c}
\rho_{1} e^{i \varphi_{1}} \\
\rho_{2} e^{i \varphi_{2}} \\
\vdots \\
\rho_{R} e^{i \varphi_{R}}
\end{array}\right], \quad \boldsymbol{\rho}=\left[\begin{array}{c}
\rho_{1} \\
\rho_{2} \\
\vdots \\
\rho_{R}
\end{array}\right], \quad \mathbf{s}=\mathbf{C} \widetilde{\boldsymbol{\rho}}=\mathbf{D} \boldsymbol{\rho}
$$

The VCC signals can be integrated by extending the signal vector with the complex conjugate signals $\mathbf{s}_{\mathrm{vcc}}=\mathbf{s}^{*}$, and the effective sensitivity matrix with its conjugate:

$$
\left[\begin{array}{c}
\mathbf{s} \\
\mathbf{s}_{\mathrm{VCC}}
\end{array}\right]=\left[\begin{array}{c}
\mathbf{D} \\
\mathbf{D}^{*}
\end{array}\right] \rho=\mathbf{D}_{\mathrm{VCC}} \boldsymbol{\rho}
$$

If the rows of $\mathbf{D}^{*}$ are linearly independent from the rows of $\mathbf{D}$, then the conditioning of the problem improves, resulting in a lower noise amplification.

Using the effective sensitivities, g-factor calculation is identical to the one in standard SENSE (1). Assuming that noise pre-whitening (21) was performed before creating VCC signals, the VCC-SENSE g-factor is given by:

$$
g_{k}=\sqrt{\left(\left(\mathbf{D}_{\mathbf{V C C}}^{H} \mathbf{D}_{\mathbf{v C C}}\right)^{-1}\right)_{k, k}\left(\mathbf{D}_{\mathbf{v C C}}^{H} \mathbf{D}_{\mathbf{v C C}}\right)_{k, k}}
$$

It follows from the above derivation that VCC-SENSE requires prior knowledge of the effective sensitivities, including the object phase. This can be calculated from a prescan, possibly of lower resolution, which may be combined with the measurement of the physical sensitivity profiles.

\section{$\underline{\text { VCC-GRAPPA }}$}

Inclusion of VCC signals to a GRAPPA-like reconstruction is described in the following. Most k-spacebased reconstructions can be formulated by a convolution over the k-space neighborhood and over all coil signals, as visualized in Supporting Figure S1a: 


$$
S_{j}\left(\mathbf{k}_{l}\right)=\sum_{m, n} w_{j, l, m, n} S_{m}\left(\mathbf{k}_{n}\right)
$$

Where $j$ and $l$ respectively index the coil and the k-space location of the missing signal, while $m$ and $n$ index the same for the measured signal. The convolution kernel $w$ usually only depends on the relative k-space position of measured and target signals, i.e. $\mathbf{k}_{l}-\mathbf{k}_{n}$, and is generally found by a least-square fit using the fully sampled k-space center. Kernel range commonly includes only the close neighborhood in $\mathrm{k}$-space (22). For a VCC reconstruction, the set of $N$ coils is extended with the conjugate coil signals from the opposite k-space location:

$$
S_{N+j}(\mathbf{k})=S_{j}^{*}(-\mathbf{k}) \quad j=1, \ldots, N
$$

After VCC signal creation, standard GRAPPA kernel fitting is performed using all the $2 \mathrm{~N}$ channels, and reconstruction is accomplished according to Eq. [6] with the coil summation performed over all $2 \mathrm{~N}$ coils, as depicted in Supporting Figure S1b.

Unlike VCC-SENSE, prior knowledge of the object phase is not required in the reconstruction. However, the autocalibration region of k-space should be acquired with unchanged sequence parameters to ensure phase consistency.
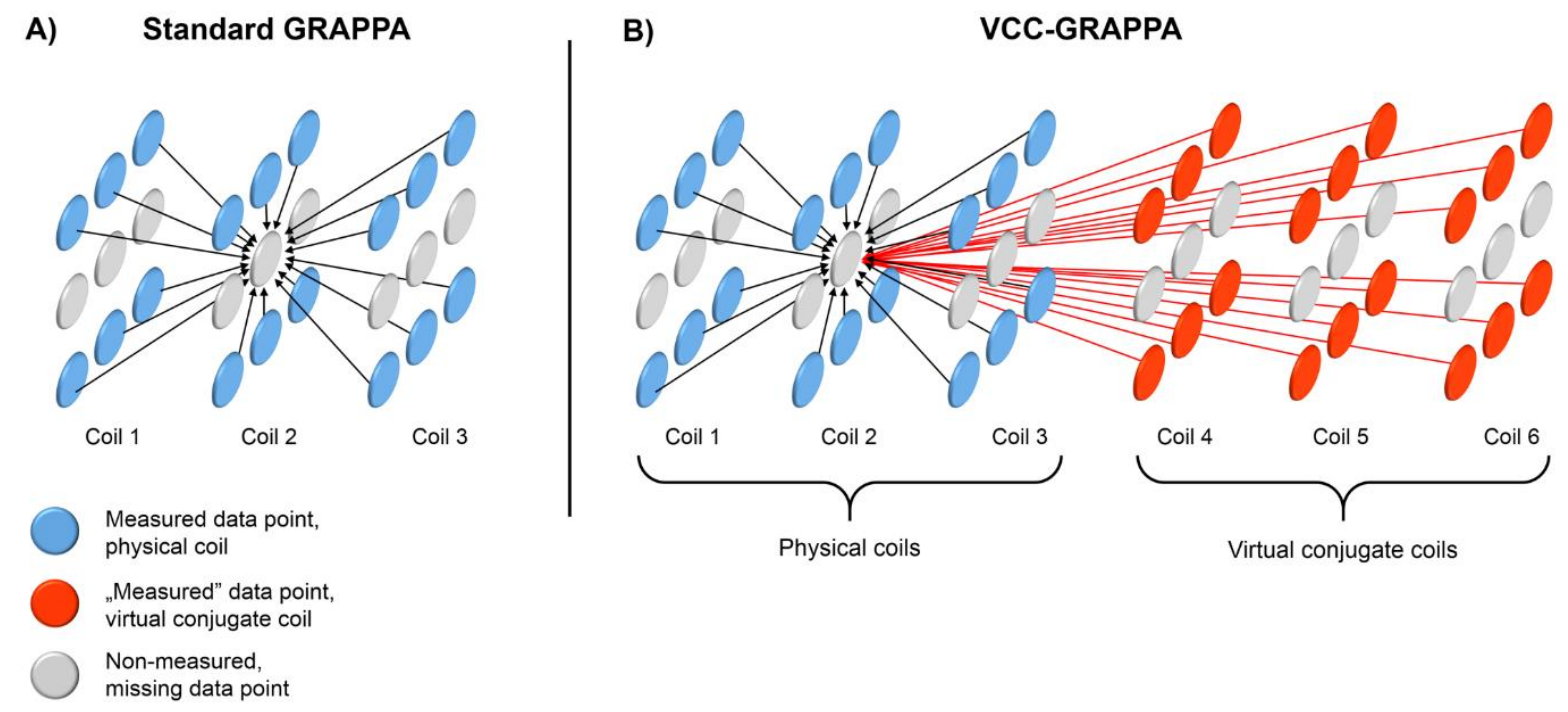

Supporting Figure S1. Schematics of standard GRAPPA and VCC-GRAPPA reconstructions for acceleration factor $\mathrm{R}=2$, using 3 coils and a kernel size of $2 \times 3$. Blue discs represent measured k-space data of physical receiver channels, orange discs represent "measured" VCC data (in reality these are calculated from measured data of physical coils), while gray discs represent missing data. Black and red arrows indicate the contribution of physical and VCC signal to the calculation of a missing data point. 


\section{Impact of background phase on noise amplification}

Here we describe the effect of the object background phase on the reconstruction, using the same notation as in Eqs. [3, 4]. With the phases of the aliasing voxels $\varphi_{1}, \varphi_{2}, \ldots, \varphi_{R}$, the elements of $\mathbf{D}_{\mathbf{V C C}}^{H} \mathbf{D}_{\mathbf{V C C}}$ will be (see Appendix 1):

$$
\left(\mathbf{D}_{\mathbf{V C C}}^{H} \mathbf{D}_{\mathbf{V C C}}\right)_{j, k}=2 \cdot \operatorname{Re}\left(\mathbf{c}_{j}^{H} \mathbf{c}_{k} \cdot e^{i\left(\varphi_{k}-\varphi_{j}\right)}\right)
$$

Where $\mathbf{c}_{k}$ is the $k$-th column of the sensitivity matrix $\mathbf{C}$, and $\operatorname{Re}(\cdot)$ is the real part operation. Using this, the VCC g-factors can be calculated using Eq. [5], and an optimal background phase set that minimizes them can be found.

Note that, for a given coil geometry, the elements of $\mathbf{D}_{\mathbf{V C C}}^{H} \mathbf{D}_{\mathbf{V C C}}$, hence also the g-factors, are only dependent on phase differences between aliasing voxels, meaning that adding a constant phase shift to all voxels in the aliasing group does not change the reconstruction quality. Moreover, this phase shift can be different for each overlapping voxel group without affecting the g-factors, as long as all voxels within a group experience the same phase shift. This allows one to choose a spatially smooth optimal phase map by carefully selecting the phase shifts added to each overlapping voxel group, as elaborated in the next section.

The minimization of g-factors means that we want to reduce or eliminate the off-diagonal elements of $\mathbf{D}_{\mathbf{V C C}}^{H} \mathbf{D}_{\mathbf{V C C}}$, since a diagonal case would mean that all g-factors in that aliasing group are equal to 1 . According to Eq. [8], this means that we want the background phase to result in a purely imaginary value

of $\mathbf{c}_{j}^{H} \mathbf{c}_{k} \cdot e^{i\left(\varphi_{k}-\varphi_{j}\right)}$. For entirely real-valued coil sensitivities, a phase difference of $\pi / 2$ between the overlapping voxels is the optimal solution and results in g-factors of 1 in the extreme case of one coil and two-fold overlapping (10). In a general setup with multiple receiver coils using acceleration factor R, g-factors of the VCC reconstruction with optimal object phase are identical to those of a conventional reconstruction with acceleration factor $\mathrm{R} / 2$. A simulation example for a gradient-echo-EPI acquisition with acceleration factor $\mathrm{R}=8$ using 52 receiver channels is displayed in Supporting Figure S2, showing that the g-factor maps of the $\mathrm{VCC}$ recon with $\mathrm{R}=8$ and the conventional recon with $\mathrm{R}=4$ are almost indistinguishable.

Intuitively, the benefit of optimal phase pattern can be thought of as an analogy of optimal coil sensitivities and aliasing pattern. In a phase-constrained reconstruction, the phase pattern works like an additional spatial encoding function, much like an additional coil, which modifies the noise amplification. As the g-factors in conventional PI can be varied by changing coil geometry, or by modifying the aliasing pattern via optimized phase-encoding patterns like in CAIPIRINHA $(23,24)$; in phase-constrained PI, 
g-factors can additionally be varied by changing the phase distribution. The benefit of phase optimization can be well illustrated in the above-mentioned extreme case of one receiver channel and two-fold acceleration. In this setup, the only information available to separate overlapping voxels is the phase difference between them. Without phase optimization, the generally small differences in the inherent object phase could result in immense noise amplification, or even render the reconstruction impossible; while introducing the optimal phase distribution can decrease the g-factors to 1 everywhere, providing a perfect reconstruction.

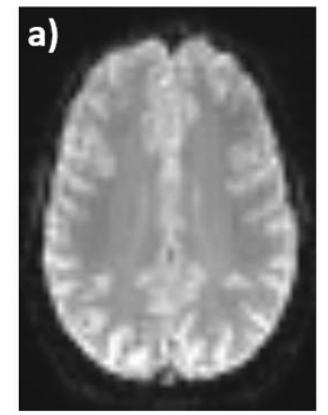

Magnitude image of GRE-EPI b)

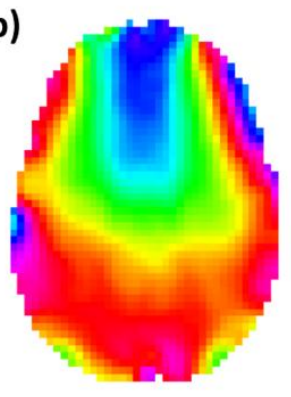

Extracted object phase c)

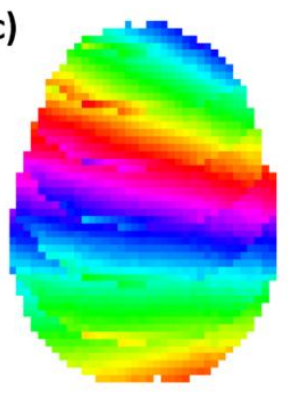

Calculated optimal phase map d)

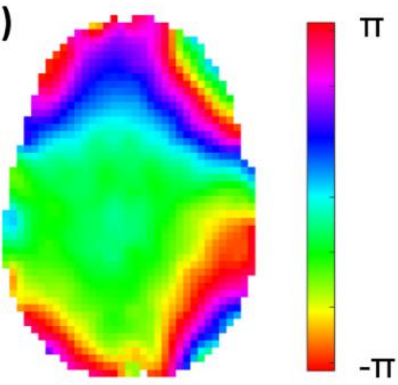

Calculated target phase map

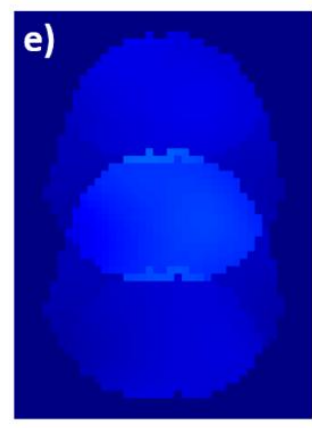

Conventional recon, $\mathrm{R}=4$

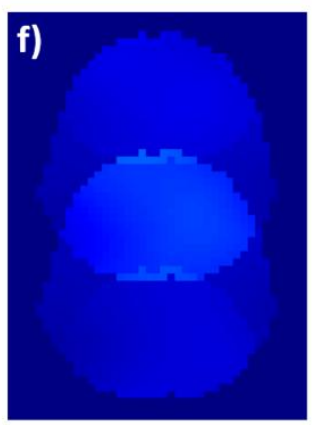

VCC recon with optimal phase map, $\mathrm{R}=8$

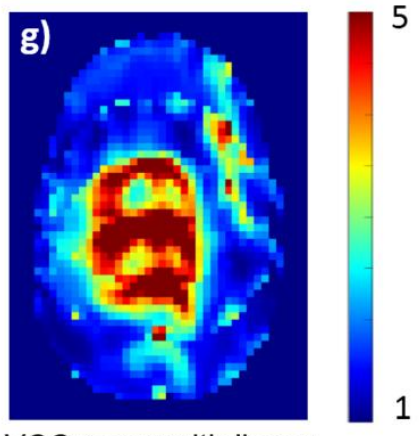

VCC recon with linear phase-ramp, $\mathrm{R}=8$

Supporting Figure S2. Exemplary calculated optimal and target phase maps and simulated g-factors for a gradient-echo-EPI acquisition, for acceleration factor $\mathrm{R}=8$. Magnitude reconstruction of the full measurement (a) is shown as well as the extracted object phase (b), the computed optimal phase map (c), and the target phase map of the RF pulse (d). Note that, the optimal phase map is quite similar to the one shown in Figure 1, as it only depends on the receiver sensitivity profiles and the acceleration design, which was the same in both cases. However, the target phase map is strongly different from the one in Figure 1, as the object phase has far more spatial variance in this GRE-EPI example.

Moreover, this target is significantly different than a simple linear ramp along PE direction, as expected due to the high intrinsic phase variations in a gradient-echo-EPI measurement. In the lower row, simulated g-factor maps are shown for conventional reconstruction with $\mathrm{R}=4$ (e), VCC reconstruction with optimal phase map with $\mathrm{R}=8$ (f), and VCC reconstruction with a linear phase-ramp with $\mathrm{R}=8(\mathrm{~g})$. g-Factors of VCC recon with optimal phase are nearly identical to the conventional recon with halved acceleration factor. Also, g-factors in the case of optimized phase map (f) are significantly lower than the ones with a simple spatially linear phase-ramp $(\mathrm{g})$. 


\section{Methods}

All experiments were performed on a 3T clinical scanner (MAGNETOM Prisma, Siemens Healthcare, Erlangen, Germany) equipped with 20-channel and 64-channel head-neck receiver arrays having 16 and 52 active head channels, respectively; and a two-channel built-in body coil for transmission used in circular polarization mode. All calculation and reconstruction was performed offline using MATLAB (The MathWorks, Natick, MA, USA). In accordance with institutional regulations, the involved volunteer gave written informed consent before the study. All measurements were performed using prototype sequences.

\section{Overview of the procedure}

To test the benefit from the optimized object phase distribution, the following procedure was performed. A detailed description of each step is presented later in this section.

1. A full-sampled prescan was performed with conventional excitation.

2. From the prescan data, coil sensitivity maps were estimated, and the intrinsic object phase map was extracted. From these, a spatially smooth optimal background phase distribution was calculated for the desired acceleration factor.

3. A tailored RF excitation pulse was designed for the optimized phase map, and

4. was applied in subsequent accelerated and full scans, with the latter serving as a gold-standard in the evaluation of the reconstruction.

Conventional and optimized accelerated measurements were reconstructed with and without the inclusion of VCC signals. Results were compared to the full-sampled gold-standard images; g-factor maps were computed for all cases. Flip angle inhomogeneity of the tailored excitations was measured by comparing conventional and optimized scans.

\section{Prescan, sensitivity estimation and phase extraction}

As can be seen from Eqs. [5, 8], the optimal object phase is highly dependent on the coil sensitivity profiles. Also, in order to determine the desired phase distribution, knowledge of the intrinsic object phase is required as it will be superimposed on any phase map introduced by a tailored excitation. For this reason, after a short noise measurement, a fully sampled prescan was performed using non-selective excitation followed by slice-selective refocusing. A non-selective excitation was chosen to make the prescan comparable to the optimized scan, where tailored excitation was also non-selective. Besides the excitation pulse, sequence parameters of the prescan were identical to the subsequent optimized acquisition. An accelerated dataset using conventional excitation was also acquired for comparison. 
Measured data were reconstructed and evaluated offline in MATLAB. Noise pre-whitening was performed on the receiver channels using the noise-only acquisition (21). Complex-valued sensitivity profiles were estimated with ESPIRiT (20) and were subsequently used to extract the intrinsic object phase distribution.

\section{Computing optimal phase distribution and target phase map}

A binary mask of voxels with relevant signal was produced from prescan images. For each overlapping voxel group in a given acceleration scheme, optimal background phase values were computed using an iterative, derivative-free simplex search algorithm (25), by minimizing the sum-of-squared g-factor values in the voxel group. Initial values for phase differences were chosen to be spatially linear in the direction of acceleration with a $\pi / 2$ difference between neighboring overlapping voxels, as this is expected to be close to the optimum (10). Voxels outside the binary masks were disregarded in the optimization procedure. The initial target phase map for the tailored RF pulse was taken as the difference of the optimal phase and the intrinsic object phase. Up to this point the procedure described above is similar to the work presented in (17). However, in our case, another simplex search was performed on the initial target to make it spatially smooth for physical feasibility. In this step, different phase shifts were added to each overlapping voxel group separately, in order to reduce discontinuities and edges in the target phase map, while maintaining optimal g-factors. Finally, the target was filtered with an isotropic Gaussian kernel of $8 \mathrm{~mm}$ standard deviation. The scheme of the whole procedure is depicted in Figure 1. 


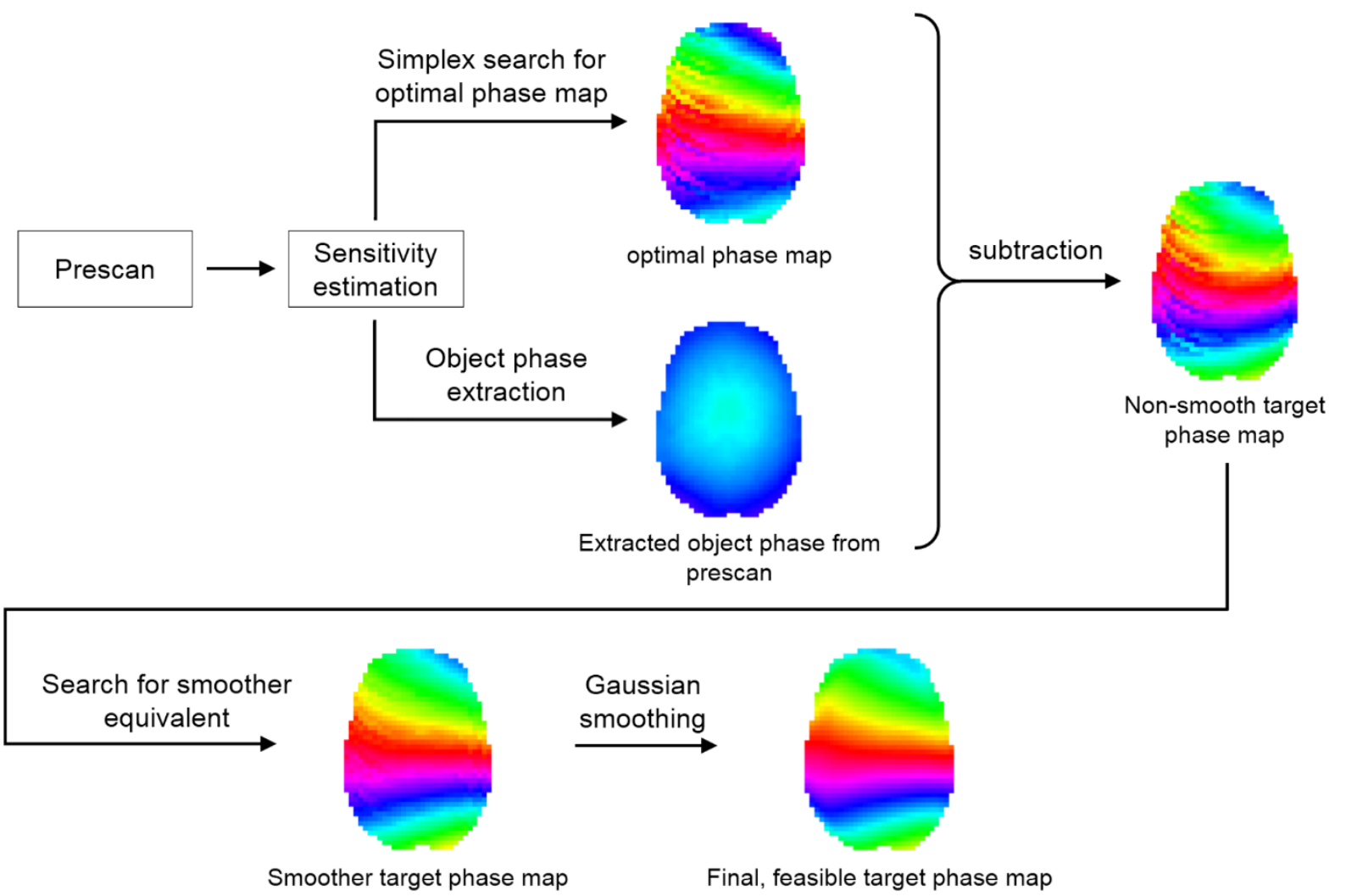

Figure 1. Scheme of the target phase map calculation procedure. As shown, the calculation of the initial target phase is followed by another simplex search to find a smoother phase distribution with unchanged SENSE g-factors, and a final Gaussian filtering to ensure the feasibility of the target. Note that, the optimal phase only depends on the receiver sensitivity profiles and the acceleration pattern, and does not depend on the object phase, therefore, it is expected to be similar to the one shown here regardless of the object being measured, provided that the imaging geometry, the receiver coil, and the acceleration pattern is the same. However, the target phase is computed as the voxel-wise difference between optimal phase and inherent object phase, causing it to depend heavily on the object and its background phase (see also Supporting Fig. S2). Source code and sample data are available for research purposes at https://github.com/adamkettinger/phase-optimization-for-VCC.

\section{$\underline{\text { RF pulse design for in-plane acceleration }}$}

Tailored RF pulses for in-plane acceleration used spiral-out gradient waveform (26) optimized for hardware limits. As the target phase pattern for spin-echo and SE-EPI shows close resemblance to a linear phase-ramp in the PE direction, the rewinder of the pulse was designed to the excitation k-space coordinate corresponding to this phase-ramp, effectively shifting the center of the spiral from k-space origin. Since an acceleration factor $\mathrm{R}=8$ was used for all in-plane cases, the spiral was centered to $k_{0}=4 \cdot \Delta k$, where $\Delta k$ is the phase-encoding sampling distance in the full scan. 
The complex RF waveform for the target phase was designed in the low-flip-angle approximation, with $6.5 \mathrm{~mm}$ excitation resolution and $4 \mathrm{~mm}$ image-space target resolution, for a single transmission coil, using the technique described in (27), solving the design equation directly. Voxels outside of the object binary mask were disregarded during pulse calculation. Resulting excitation pulses of length $8.12 \mathrm{~ms}$ were subsequently applied in the optimized scan.

\section{$\underline{\text { RF pulse design for SMS acceleration }}$}

Tailored RF pulses for SMS acquisition used stack-of-spirals gradient waveform, designed using the same script (26), with the number of spirals being equal to the number of simultaneously measured slices, which was 3 in all SMS cases. To decrease pulse length, 3D tailored pulses had a lower in-plane excitation resolution of $10 \mathrm{~mm}$, allowed by the smoother target phase distribution (see Supporting Figure S4, top right). RF waveform calculation was performed similarly as for in-plane acceleration, resulting in a pulse length of $13 \mathrm{~ms}$. Exemplary pulses and phase targets are shown in Supporting Figures S3 (in-plane) and S4 (SMS).

Note that, while $\mathrm{B}_{1}$ inhomogeneity plays a substantial role in the intrinsic object phase, it will not affect the phase of the optimized scan, since the target is defined as the difference of optimal and intrinsic phases (derivation in Appendix 2).
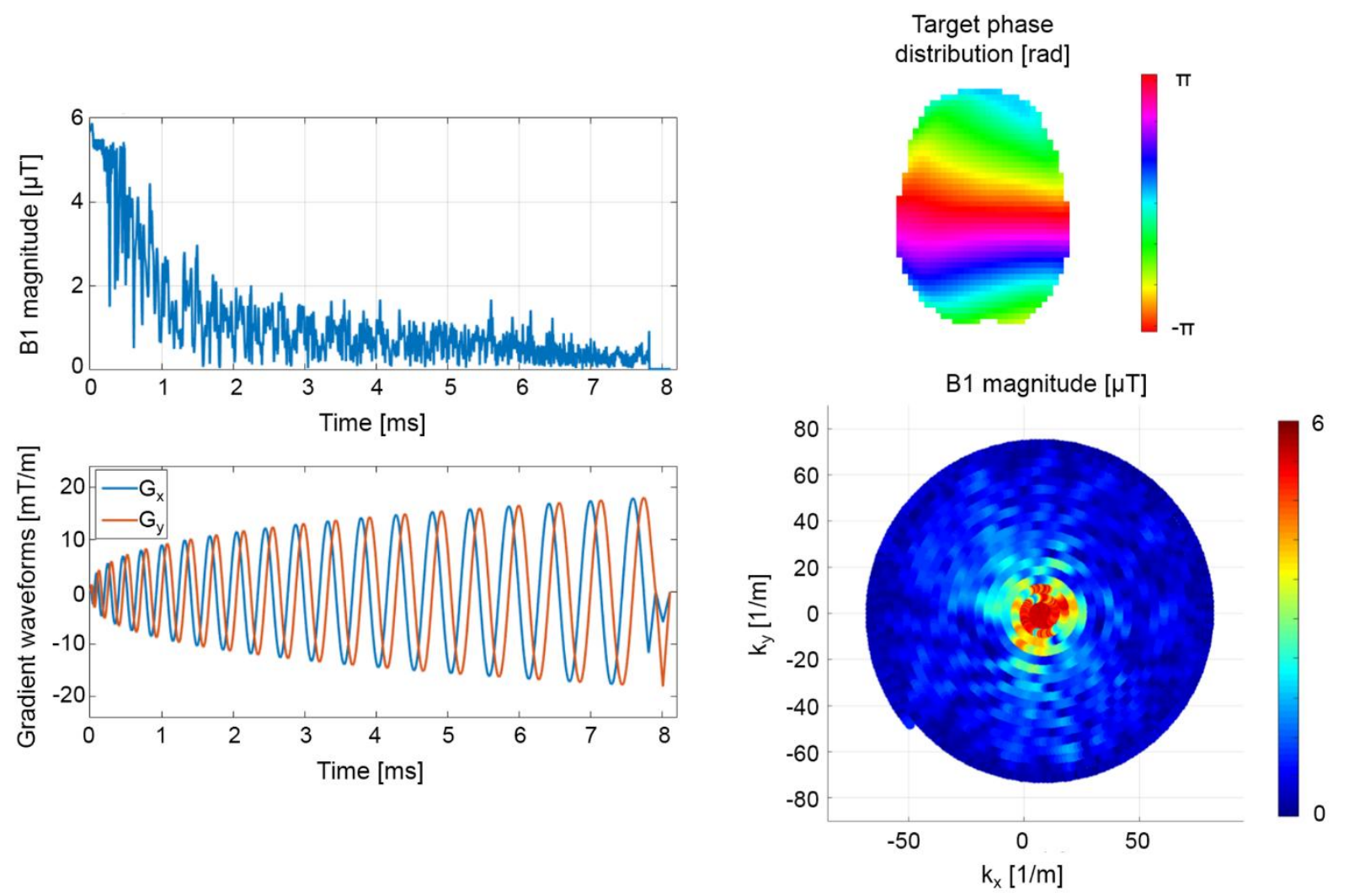
Supporting Figure S3. Exemplary target phase profile and waveforms for tailored 2D excitation for acceleration factor 8. RF pulse magnitude (top left) and spiral gradient waveforms (bottom left) are shown as the function of time. RF magnitude as the function of excitation k-space (bottom right) and corresponding target phase profile (top right) are displayed. Note that, the spiral is not centered to k-space origin, but slightly shifted in the phase-encoding direction ( $\mathrm{kx}$ in the figure). The center of the spiral corresponds to the spatially linear phase-ramp in phase-encoding direction which introduces $\pi / 2$ phase difference between neighboring overlapping voxels.
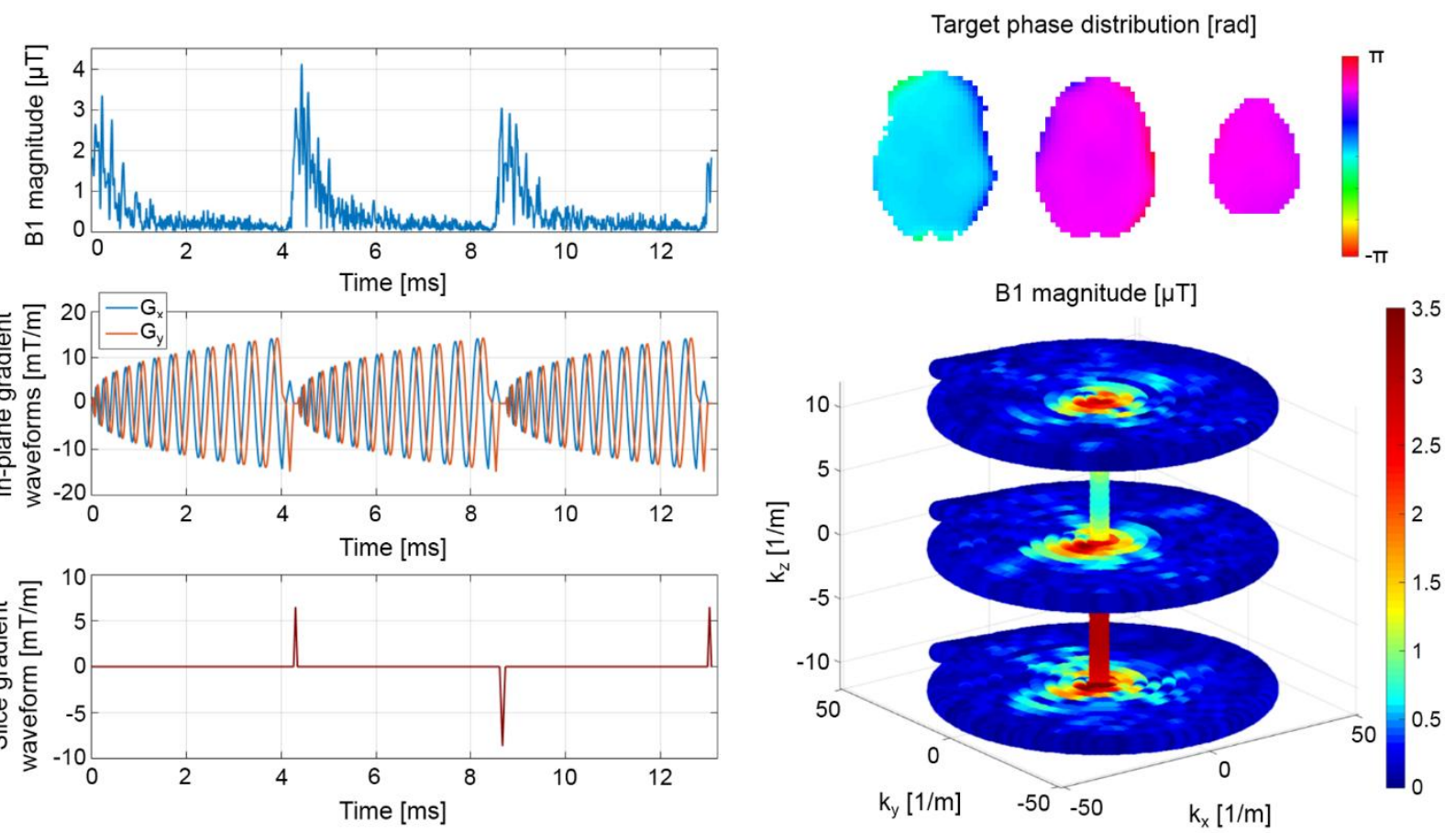

Supporting Figure S4. Target phase profile and waveforms for tailored 3D excitation pulse for SMS acceleration factor 3. RF pulse magnitude (top left), in-plane gradient waveforms (middle left) and slice gradient waveform (bottom left) are shown as the function of time. RF magnitude as the function of excitation k-space (bottom right) and corresponding target phase distribution (top right) are also displayed.

\section{$\underline{\text { Measured and simulated datasets }}$}

To test the method, the following datasets were acquired and analyzed in the subsequent reconstruction. In each case, accelerated data and full measurements were produced.

- Measured data using conventional excitation

- Measured data using tailored excitation

- Simulated data using conventional excitation and spatially linear phase-ramp

- Simulated data using tailored excitation and synthetically generated ACS data 


\section{$\underline{\text { Reconstruction }}$}

After noise pre-whitening, all in-plane-accelerated dataset were reconstructed using the iGRAPPA algorithm (28) to iteratively increase the calibration region, while performing a standard GRAPPA in each step. Kernel sizes of 2x9 and 16x7 were used for in-plane PI and SMS acquisitions, respectively. Note that, although a kernel using 16 PE lines seems large, in fact the actual PE range covered by the kernel did not change from in-plane PI to SMS, as the spacing of measured PE lines decreased by a factor of 8 . The final magnitude image was computed as a sum-of-squares combination of the reconstructed coil images. For each accelerated dataset, a VCC-iGRAPPA reconstruction was also performed, by extending the receiver set with the VCC signals before kernel fitting. Autocalibration lines were extracted from the full measurements, and were not included in the final reconstruction step. 24 and 32 ACS lines were used for the EPI and the T1-weighted spin-echo measurements, respectively.

In this work, the GRAPPA kernel is estimated from the ACS acquired with tailored excitation. However, acquiring the prescan with conventional excitation and additional ACS lines with tailored excitation is time-consuming. Therefore, we additionally investigated the possibility of training the GRAPPA kernel for the optimized scan using only the prescan. The training dataset was computed by multiplying the conventional scan dataset in image-space with the expected phase distribution resulting from the tailored excitation, which was calculated by a numerical Bloch simulation of the designed RF pulse (29). Such calculation of the kernel may eliminate the need of measuring ACS lines in the optimized scan, and thus reduce measurement time even further.

SMS acquisitions were also reconstructed with iGRAPPA using the SENSE/GRAPPA hybrid method $(16,18)$. In this case, the full measurement was used for kernel calibration, as SMS EPI experiments usually perform a full prescan before the accelerated acquisition.

Reconstructed magnitude images were afterwards compared to each other and to the full measurements. g-Factor maps were calculated from GRAPPA kernels as described in (3). Differences between accelerated and full measurements were computed to investigate reconstruction quality and remaining artifacts. Differences between full measurements of conventional and tailored excitations were also calculated to visualize flip angle inhomogeneity of the tailored pulses. Standard deviation of this voxelwise difference was divided by the mean intensity of the conventional scan; the result was taken as a measure of flip angle inhomogeneity. In all calculations, only voxels within the object binary mask were considered.

\section{$\underline{\text { In-vivo measurements }}$}


Designed RF pulses were tested in vivo on a volunteer's brain. Prototype T1-weighted spin-echo and segmented SE-EPI sequences were used in the case of in-plane acceleration, while SMS measurements were performed using single-shot SE-EPI. Relevant sequence parameters are summarized in Table 1.

In the spin-echo acquisitions, accelerated datasets were simulated by extracting a fraction of the phaseencoding lines. In segmented SE-EPI, the number of interleaved segments coincided with the desired acceleration factor $\mathrm{R}=8$; the accelerated dataset was taken as the single segment containing the $\mathrm{k}$-space center line. In the SMS experiments, an accelerated acquisition was performed, that is, after a full measurement where all slices were measured separately, a subsequent accelerated scan was performed measuring all 3 slices simultaneously using multiband refocusing pulses. No in-plane acceleration was used in the SMS scans.

\begin{tabular}{|c|c|c|c|}
\hline & \multicolumn{2}{|c|}{ In-plane acceleration } & \multirow{2}{*}{$\begin{array}{c}\begin{array}{c}\text { simultaneous } \\
\text { multi-slice (SMS) } \\
\text { acceleration }\end{array} \\
\text { single-shot spin-echo } \\
\text { EPI }\end{array}$} \\
\hline & T1-weighted spin-echo & segmented spin-echo EPI & \\
\hline Excitation type & nonselective / tailored & nonselective / tailored & nonselective / tailored \\
\hline Excitation flip angle & $20^{\circ}$ & $15^{\circ}$ & $15^{\circ}$ \\
\hline Refocusing type & single slice selection & single slice selection & single slice / multiband \\
\hline $\begin{array}{r}\text { Refocusing flip } \\
\text { angle }\end{array}$ & $180^{\circ}$ & $180^{\circ}$ & $180^{\circ}$ \\
\hline Repetition time (TR) & $900 \mathrm{~ms}$ & $5000 \mathrm{~ms}$ & $5000 \mathrm{~ms}$ \\
\hline Echo time (TE) & $25 \mathrm{~ms}$ & $50 \mathrm{~ms}$ & $85 \mathrm{~ms}$ \\
\hline slice thickness & $6 \mathrm{~mm}$ & $6 \mathrm{~mm}$ & $5 \mathrm{~mm}$ \\
\hline in-plane resolution & $1.33 \mathrm{~mm}$ & $2 \mathrm{~mm}$ & $2 \mathrm{~mm}$ \\
\hline in-plane matrix size & $192 \times 192$ & $128 \times 128$ & $128 \times 128$ \\
\hline $\begin{array}{r}\text { phase-encoding } \\
\text { direction }\end{array}$ & AP & AP & AP \\
\hline $\begin{array}{r}\text { receiver channels } \\
\text { used }\end{array}$ & 52 & 52 & 16 \\
\hline $\begin{array}{r}\text { SMS acceleration } \\
\text { factor }\end{array}$ & 1 (no SMS) & 1 (no SMS) & 3 \\
\hline
\end{tabular}




\begin{tabular}{rccc}
\hline $\begin{array}{r}\text { in-plane acceleration } \\
\text { factor }\end{array}$ & 8 & 8 & 1 (no in-plane acc.) \\
\hline $\begin{array}{r}\text { distance between } \\
\text { centers of } \\
\text { simultaneously } \\
\text { measured slices }\end{array}$ & $-($ no SMS) & - (no SMS) & $30 \mathrm{~mm}$ \\
\hline number of segments & - & 8 & - \\
\hline fat saturation & used & used & used \\
\hline Accelerated dataset & $\begin{array}{c}\text { simulated by } \\
\text { extracting every } 8^{\text {th }} \\
\text { line }\end{array}$ & $\begin{array}{c}\text { simulated by taking the } \\
\text { segment containing the } \\
\text { k-space center line }\end{array}$ & $\begin{array}{c}\text { measured using } \\
\text { multiband refocusing }\end{array}$ \\
\hline
\end{tabular}

Table 1. Protocol parameters for the used prototype sequences. The parameters changing between conventional and optimized scans are separated by a slash within a cell.

\section{Results}

$\underline{\text { Reduction of noise amplification }}$

Reconstructed magnitude images and calculated g-factor maps of the in-plane accelerated SE-EPI and T1-weighted spin-echo measurements are shown in Figures 2 and 3, respectively. It can be seen that just including the VCC signals into the reconstruction affects the image quality only slightly, as the g-factor maps show little reduction, while magnitude images are highly similar to the ones resulting from conventional reconstruction. In contrast, optimization of the object phase does have a great impact: the magnitude images show significantly reduced noise in this case. Compared to conventional reconstruction, mean geometry factors of the optimized scans are reduced by a factor of 2 . In the T1-weighted spin-echo experiments, a simple linear phase-ramp provides similar benefit to the noise amplification; however, it becomes suboptimal in EPI scans.

The results of generating synthetic ACS data and applying them to the optimized scan are displayed in Figure 4. Magnitude images are almost indistinguishable from the ones resulting from the standalone optimized scan, with g-factors of the two methods also being very similar.

Reconstructed magnitude images and calculated g-factors for SMS acquisitions are shown in Figures 5 and 6, respectively. In this case, including the VCC signals provides a large benefit in itself, due to the high variations of either object phase or receiver phases between slices. Nevertheless, the optimization of background phase outperforms every other setup. The increased g-factors in the anterior part are probably caused by the discrepancy between actual pulse result and phase target (see Supporting Figure S5), induced by the high $\mathrm{B}_{0}$ inhomogeneity in this region. It is noteworthy that, the linear phase increment 
along the slice direction produces worse results than the sole inclusion of VCC signals. This effect is caused by the intrinsic object phase, which happened to be close to a slice-wise ramp in this specific case, and was spoiled by the additional phase-ramp.

Magnitude images from the optimized setup are fairly close to the full measurements in the case of SMS acceleration. However, this could not happen for in-plane PI: even if g-factors would be 1 everywhere, the accelerated scans would still be noisier than the fully sampled ones due to the intrinsic SNR loss caused by the reduced amount of acquired data.

For better visualization of the improvements in optimized scans, larger reconstructed images are displayed in Figure 7. In the SMS case (top row), small anatomical details pointed out by arrows are barely visible and covered by noise in the conventional setup, with minor improvements in the conventional VCC case, but become more prominent and observable in the VCC-reconstructed optimized acquisition. For in-plane acceleration (bottom row), SNR is evidently increased in the optimized measurement, especially in the central area, while the conventional VCC reconstruction shows no visible SNR improvement compared to the standard reconstruction.
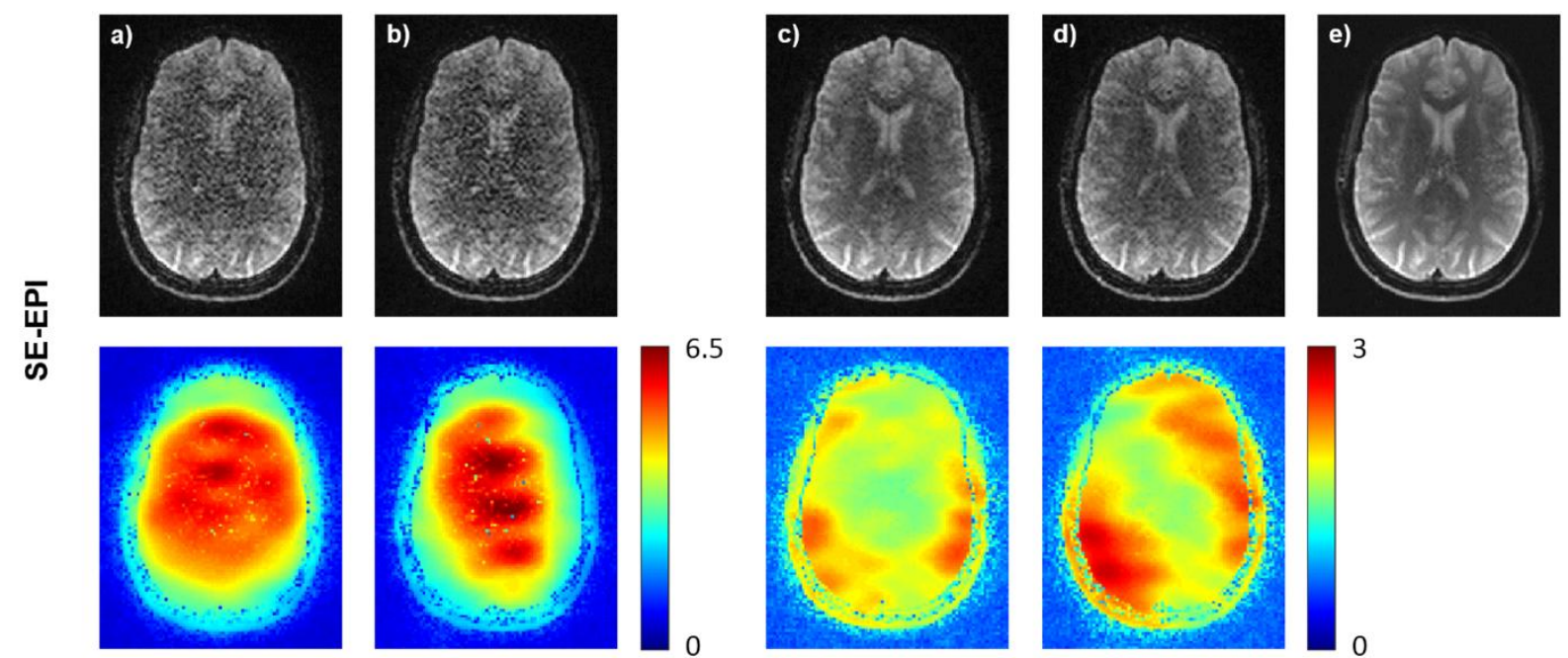

$g_{\text {mean }}$ :

4.1

3.9

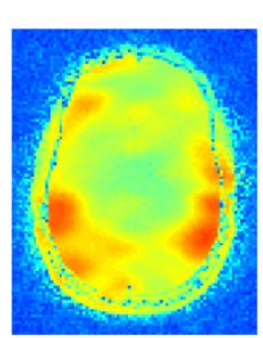

1.7

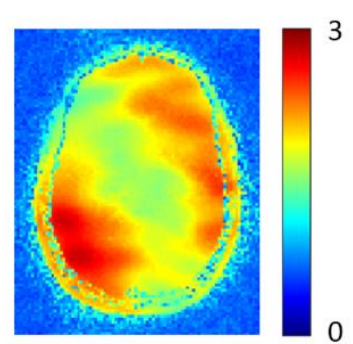

1.9

Figure 2. Reconstructed sum-of-squares magnitude images (top row), and calculated g-factors (bottom row) of SE-EPI acquisitions performed on a volunteer's brain with in-plane acceleration factor 8, using 52 receiver channels, with several measurement and reconstruction setups. Conventional scan with standard (non-VCC) reconstruction (a), conventional scan with VCC reconstruction (b), optimized scan with VCC reconstruction (c), VCC reconstruction on the simulated dataset with the spatially linear phase-ramp (d), and reconstruction of the fully sampled optimized scan (e) are displayed. 


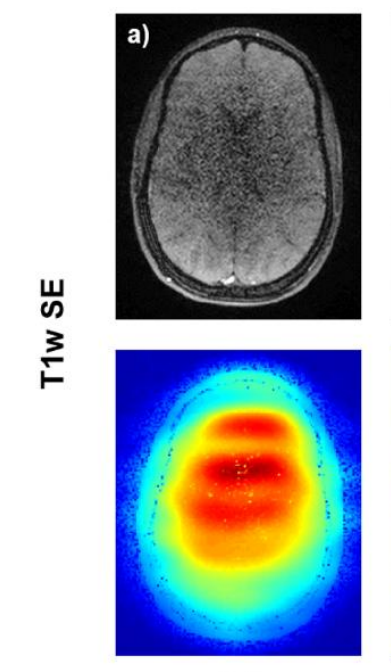

$g_{\text {mean }}$ :
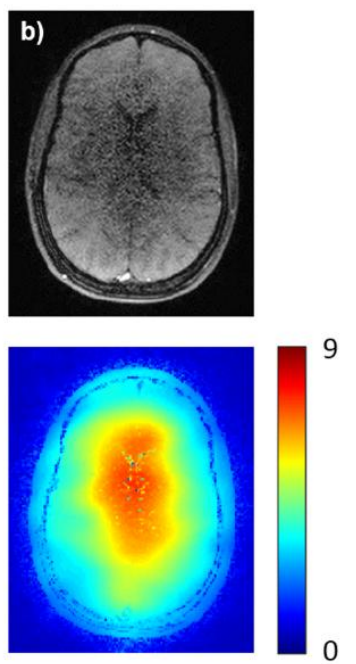

4.2
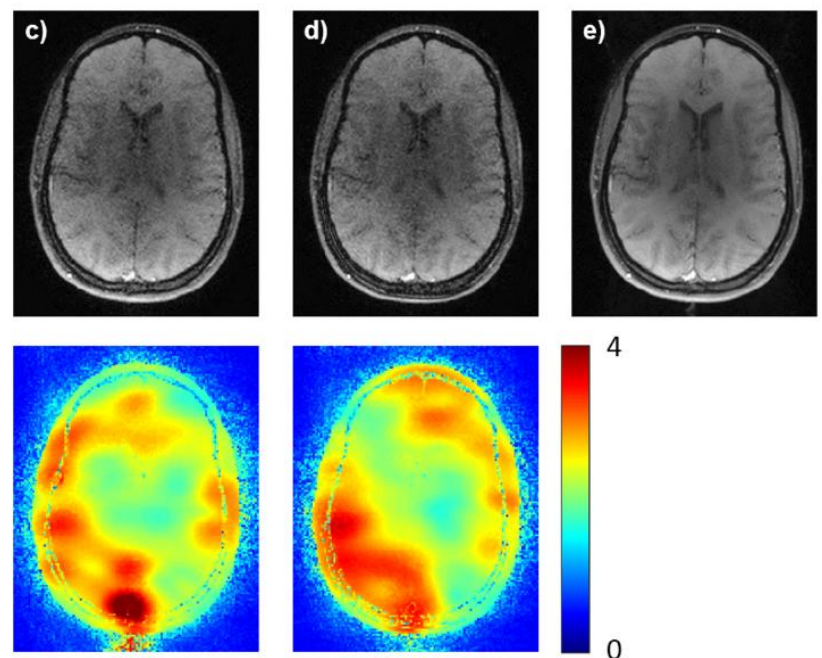

2.4

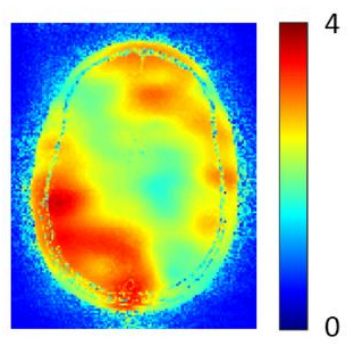

2.5

Figure 3. Reconstructed sum-of-squares magnitude images (top row), and calculated g factors (bottom row) of T1 weighted spin echo acquisitions performed on a volunteer's brain with in-plane acceleration factor 8 , using 52 receiver channels, with several measurement and reconstruction setups. Conventional scan with standard (non VCC) reconstruction (a), conventional scan with VCC reconstruction (b), optimized scan with VCC reconstruction (c), VCC reconstruction on the simulated dataset with the spatially linear phase-ramp (d), and reconstruction of the fully sampled optimized scan (e) are displayed.

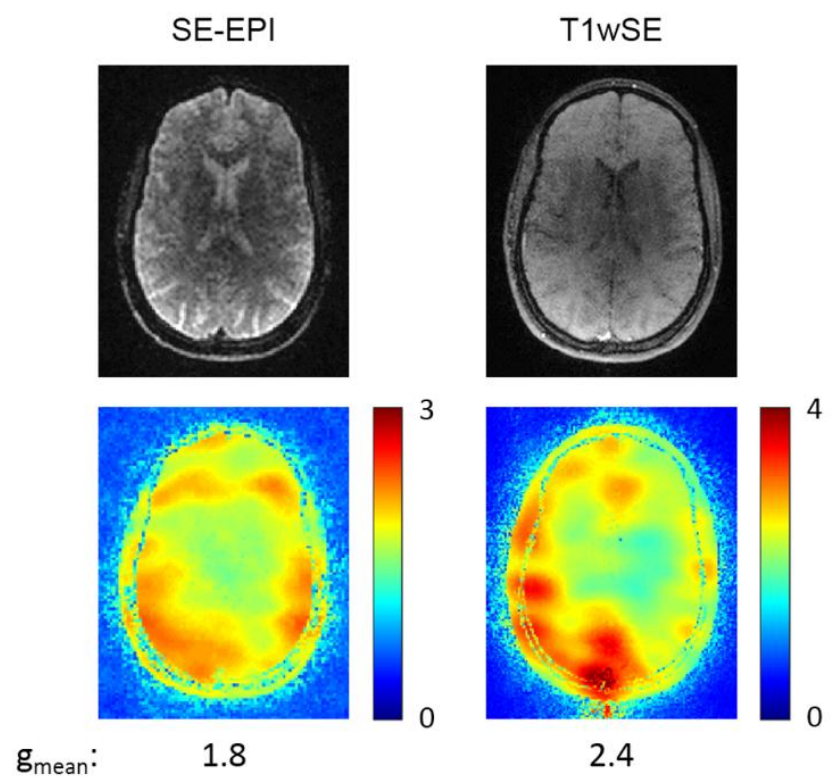

Figure 4. Reconstructed sum-of-squares magnitude images (top row) and calculated g factors (bottom row) of SE EPI (left) and T1 weighted spin echo (right) acquisitions, with in-plane acceleration factor 8, using 52 receiver channels. Tailored pulses were used for excitation. VCC reconstruction was performed using the synthetic ACS lines from the dataset simulated using the conventional scan and the target phase profile. g-Factor maps are scaled identically to the respective results shown in Figures 2 and 3. 

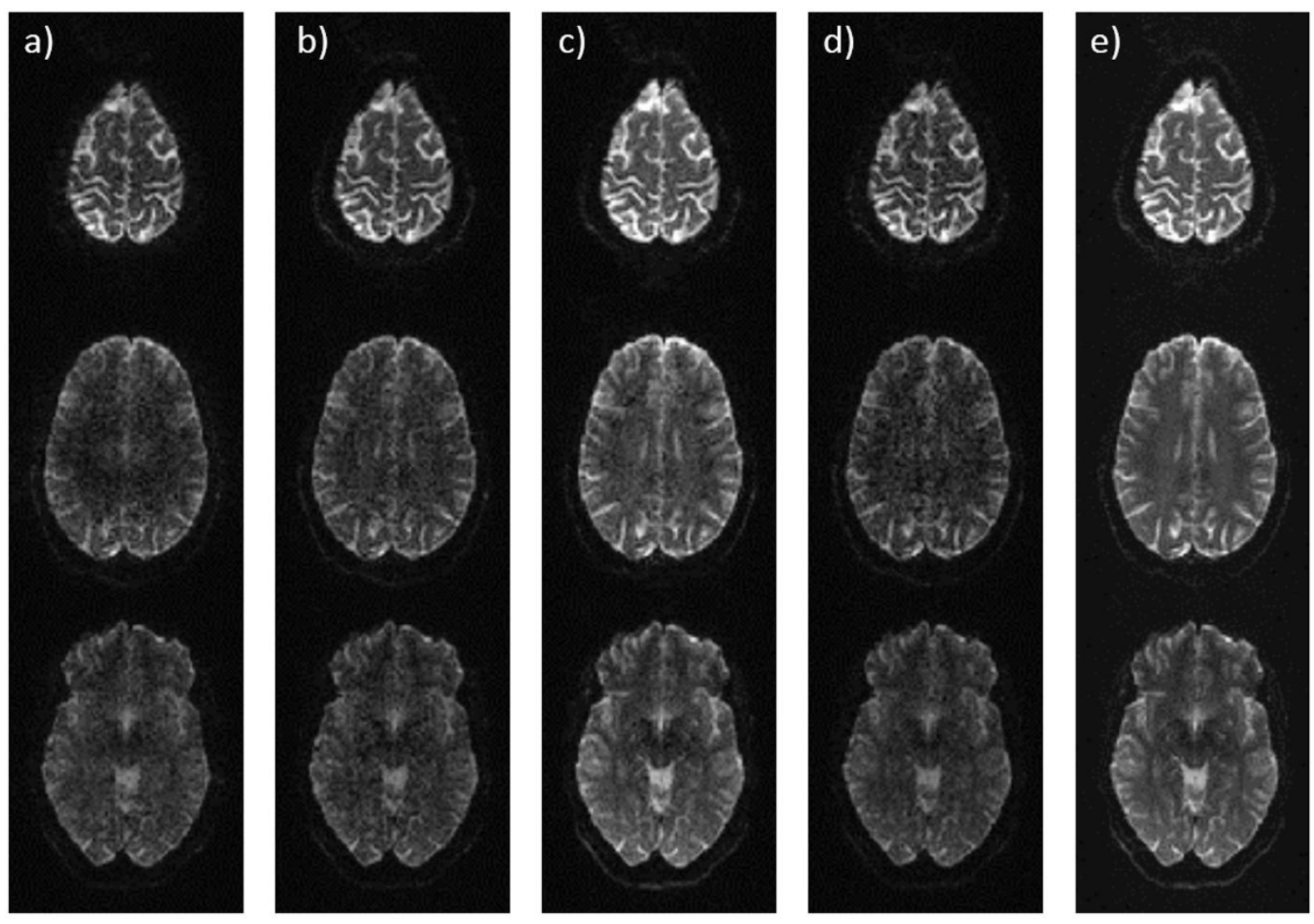

Figure 5. Reconstructed sum-of-squares magnitude images of SMS EPI acquisitions performed on a volunteer's brain with SMS acceleration factor 3, using 16 receiver channels, with several measurement and reconstruction setups. Conventional scan with standard (non VCC) reconstruction (a), conventional scan with VCC reconstruction (b), optimized scan with VCC reconstruction (c), VCC reconstruction on the simulated dataset with the spatially linear phase-ramp (d), and reconstruction of the fully sampled optimized scan (e) are displayed. 


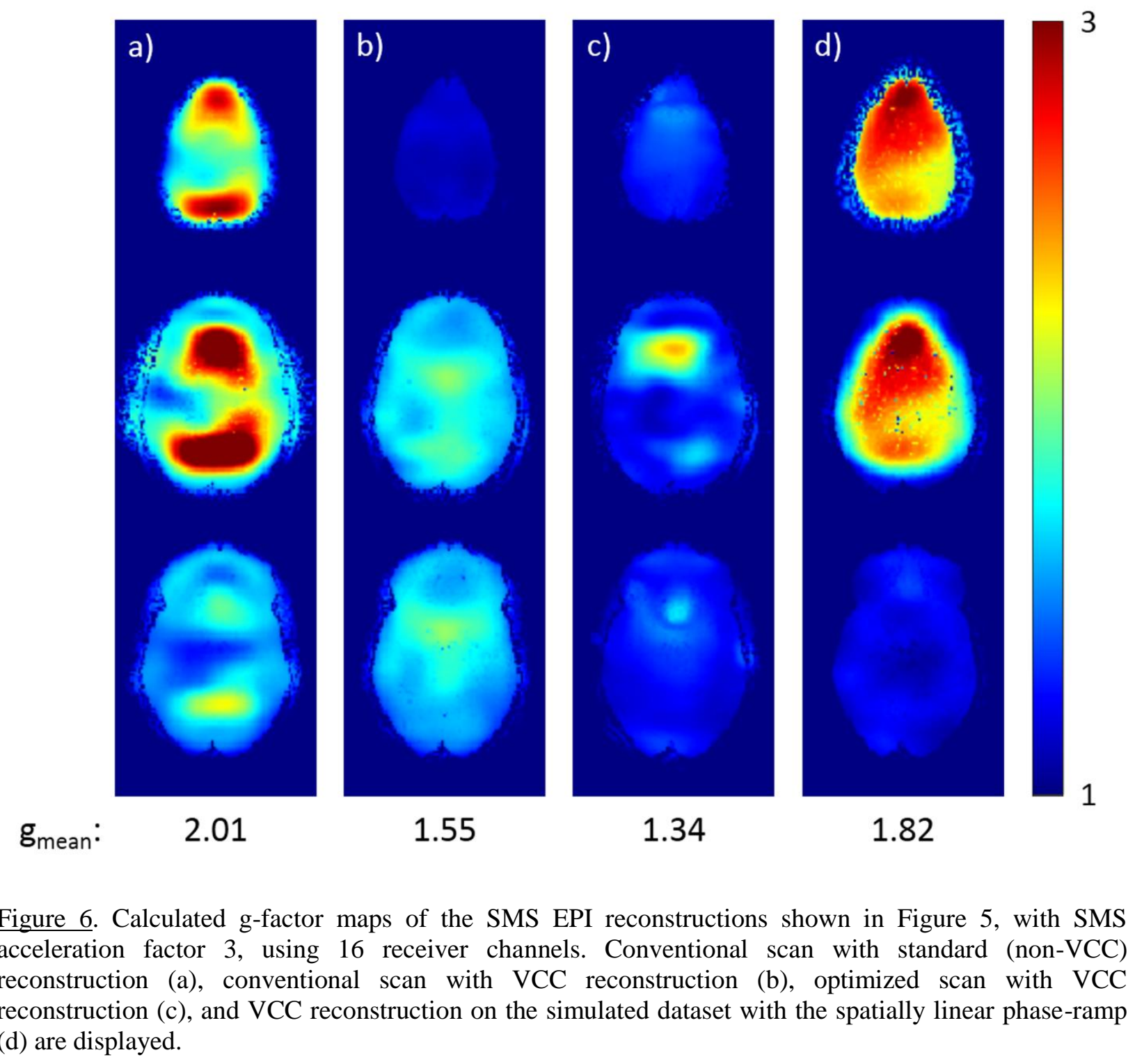



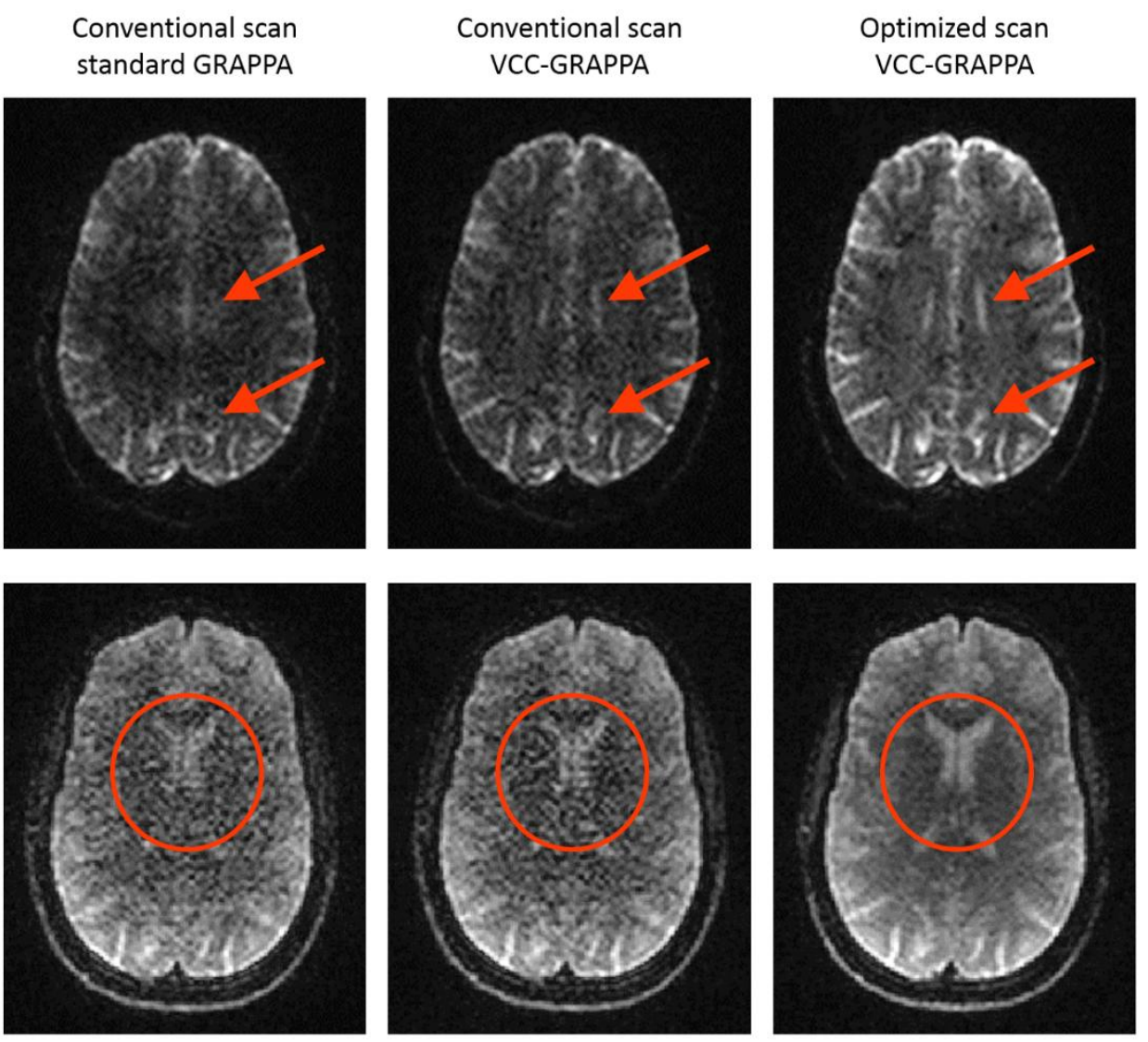

Figure 7. Enlarged reconstructions of SMS EPI (top row) and in-plane accelerated SE-EPI (bottom row) showing the benefit of VCC-reconstructed optimized scans. Small-scale anatomical details (top row, pointed out by the orange arrows) that are barely visible and covered by heavy noise in the conventional setup (left column) and in the VCC reconstruction of the non-optimized scan (middle column), become more prominent and observable in the VCC-reconstructed optimized scan (right). In the bottom row, apparent SNR in the indicated areas is similarly low in either conventional setup and in the VCCreconstructed non-optimized scan, whereas it is evidently increased in the VCC-reconstructed optimized scan. 


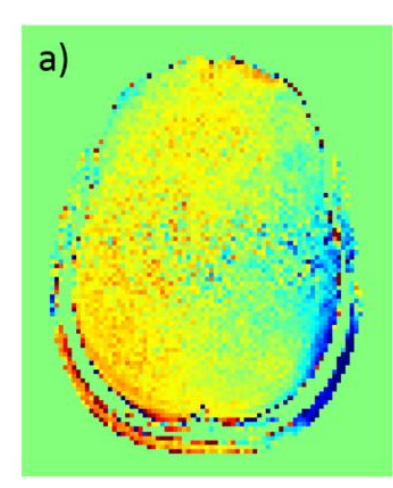

SE-EPI
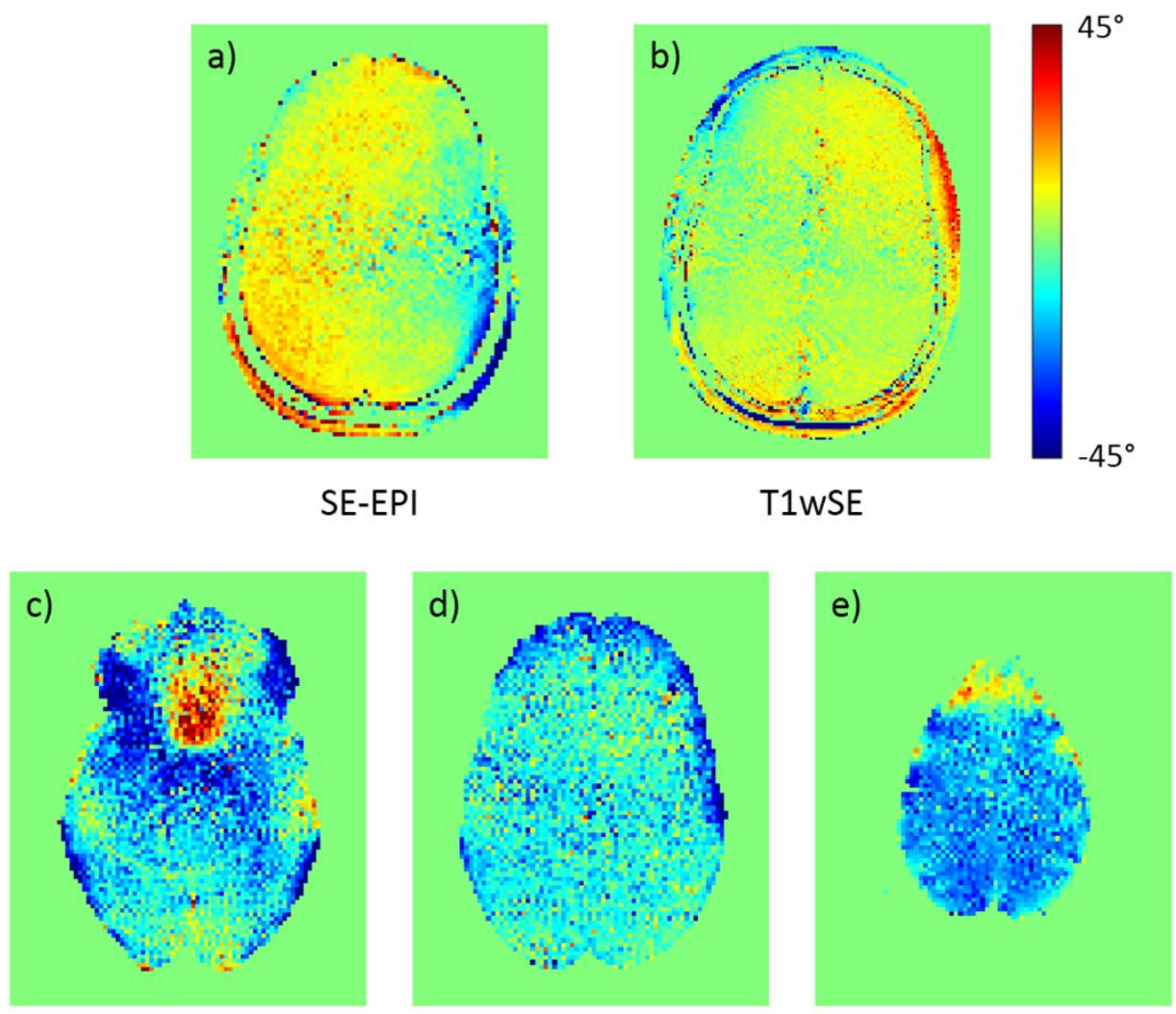

SMS EPI

Supporting Figure S5. Phase differences between the optimized measurements and the calculated targeted phase maps for SE-EPI (a), T1-weighted spin-echo (b), and SMS EPI (c-e). Theoretical phase maps were computed by adding the target phase map of the tailored RF pulse to the phase of the conventional scan.

\section{$\underline{\text { Reconstruction fidelity }}$}

To further assess reconstruction quality and visualize possible artifacts and SNR, magnitude differences between accelerated and full measurements for the conventional and optimized scans are shown in Figure 8, with 10x amplification. In the case of in-plane acceleration, little to no coherent artifact is apparent in the EPI acquisitions, with the difference being noise-like. The artifact visible in the spin-echo measurements originates from blood flow and from the Nyquist-ghost appearing in the full measurement, probably caused by some imperfections in the RF sign alternation during the acquisition. In addition, the reduced amplitude of the noise-like difference clearly indicates the increased SNR of the optimized measurements in both in-plane cases, as expected from their lower g-factors.

In the SMS experiments, although only little structured artifact is present, the overall signal intensity of the central slice is increased. This effect is independent of the phase optimization procedure, and is caused by the imperfection of the multiband refocusing pulse which produces slightly different flip angles in the 
central slices, causing this variance between full measurements (without multiband pulses) and accelerated scans. The effect of reduced difference amplitude in optimized scans is less prominent in the SMS acquisitions, probably because the benefit of phase optimization compared to standalone VCC is much lower in this case.

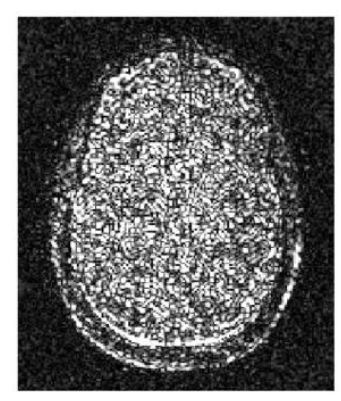

SE-EPI

Conventional scans

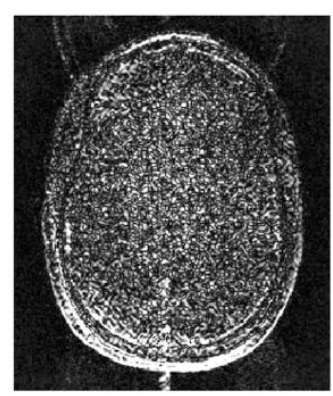

T1WSE

Conventional scans

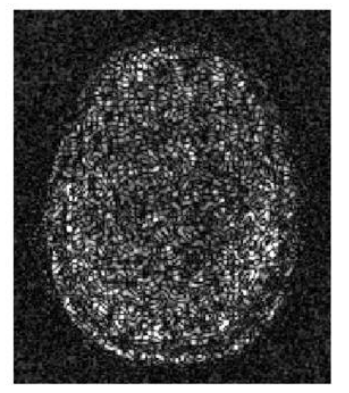

SE-EPI

Optimized scans

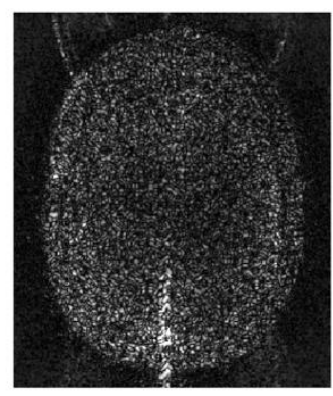

T1WSE

Optimized scans

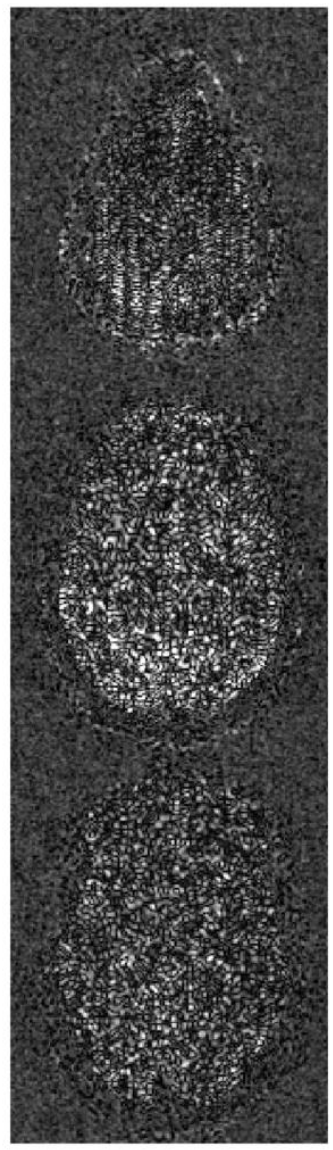

SMS EPI

Conventional scans

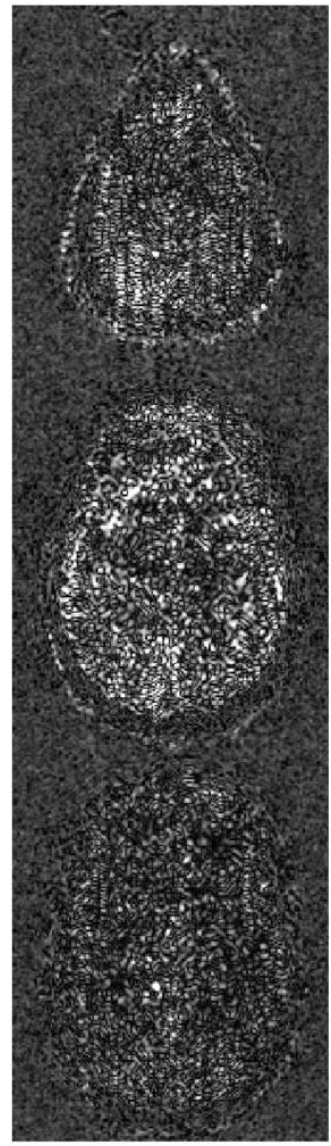

SMS EPI

Optimized scans

Figure 8. Difference images of VCC-reconstructed accelerated scans and fully sampled measurements with 10x amplification, for both optimized and conventional cases, using the same windowing as in Figures 2, 3, and 5, to visualize reconstruction fidelity, SNR, and possible remaining artifacts. The decreased amplitude of the noise-like differences in the optimized scans indicate a clear improvement in apparent SNR for the in-plane accelerations, as expected from the 2-fold g-factor reduction shown in Figures 2 and 3. This effect is not prominent for SMS EPI, as the benefit of tailored pulses compared to the standalone VCC reconstruction is lower in that case, as can be seen in Figure 6. 


\section{Flip angle homogeneity}

To visualize flip angle inhomogeneity of the tailored excitation, 5x amplified magnitude differences between conventional and optimized full measurements are shown in Figure 9. They indicate reasonably homogeneous excitation profiles of the tailored RF pulses in all cases. The relative standard deviation of the difference is around $9-13 \%$ for in-plane acceleration, and $18 \%$ for SMS measurements. Note that here we compare full measurements, which did not use multiband refocusing, hence an overall signal intensity deviation is not present.

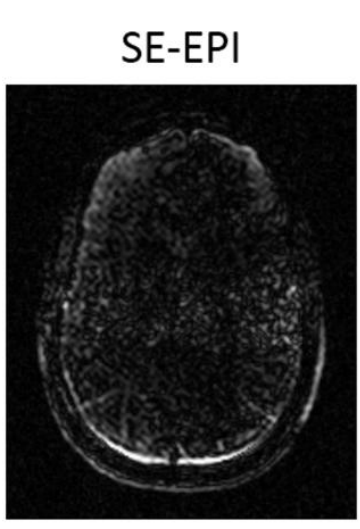

Rel. SD = $9 \%$

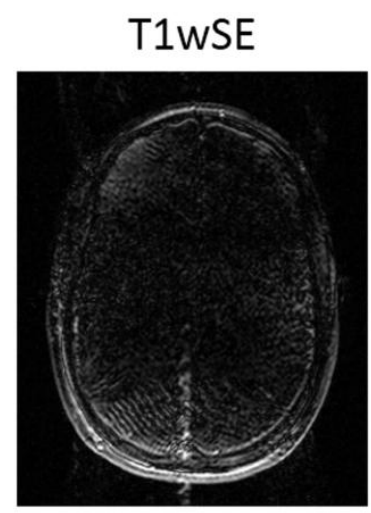

Rel. SD = $13 \%$
SMS-EPI

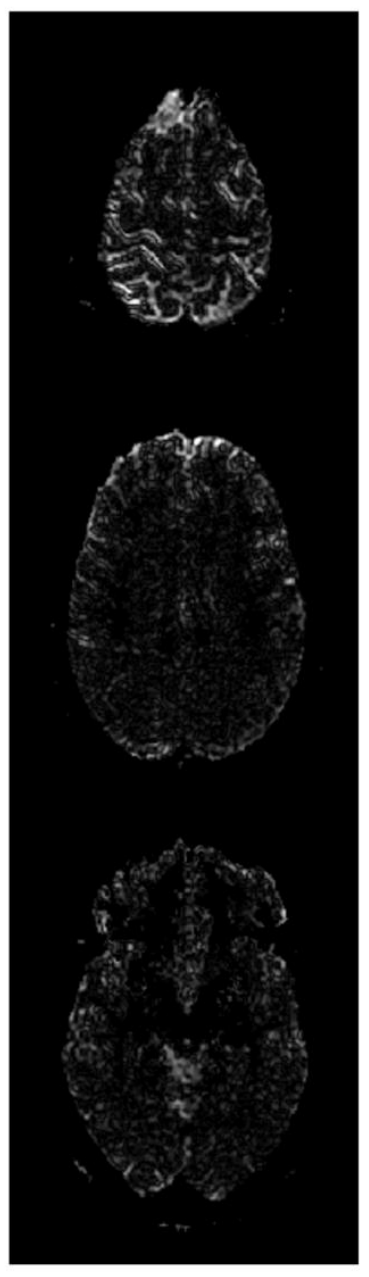

Rel. SD $=18 \%$

Figure 9. Difference images of fully sampled conventional and optimized scans with 5x amplification for the visualization of flip angle inhomogeneity of the tailored excitation, using the same windowing as in Figures 2, 3, and 5 . 


\section{Discussion}

In this work, we have demonstrated the use of tailored excitation pulses to improve the reconstruction of in-plane-accelerated as well as SMS measurements by controlling the phase distribution of the imaged object and applying phase-constrained reconstruction.

Previous work in this topic on a clinical scanner approximated the optimal phase map with a spatially linear phase-ramp achieved by an appropriate offset in k-space. Although this method has a great benefit in most cases of VCC reconstruction, we have shown that introducing the optimized, spatially non-linear phase profile can provide further improvement in reconstruction quality, especially in echo train sequences (e.g. EPI) or any other case where intrinsic phase variations are higher (see Supporting Figure S2).

Note that, our in-plane results show only minor benefits for conventional VCC reconstruction, probably caused by the small intrinsic phase variations in spin-echo-based acquisitions, and by the sensitivity of VCC to inconsistencies in k-space (movement, flow, relaxation, etc.) that are typically present in EPI measurements, degrading the VCC result and increasing g-factors locally. However, this effect is more than compensated with the optimal phase distribution.

We have also shown the possibility of training the GRAPPA kernels from a synthetically generated ACS dataset by combining optimized phase patterns with the prescan data from the conventional excitation. Results of this method are similar to the conventional technique of training the kernel using ACS from the optimized scan.

The application of phase optimization and phase-constrained reconstruction to SMS imaging was recently proposed (16); however, it was limited to introducing separate phase offsets to each slice, without considering in-plane phase variations. Using our method, it is possible to introduce the optimal phase distribution for each slice, thus decreasing the g-factors even further. Although SMS experiments in this work were performed without in-plane shifts, the principle of phase optimization is applicable for acquisitions using CAIPIRINHA $(23,24)$ or blipped-CAIPI $(6)$ to provide additional noise reduction.

Our approach requires a prescan to determine the intrinsic background phase variations as well as to estimate coil sensitivity profiles. In our work, the parameters for the prescan were identical to those of the subsequent optimized measurements except for the excitation pulse. However, in real applications the prescan is expected to have lower resolution, since phase variations and sensitivity profiles are usually spatially smooth.

Following the prescan and sensitivity estimation, an optimal phase map is numerically calculated for the given setup by minimizing the SENSE g-factors. It is currently not known whether an analytical solution 
exists; however, the overall procedure takes less than two minutes on a standard PC, and could be further accelerated by e.g. $\mathrm{C}++$ implementation or GPU computation. Note that, the algorithm minimizes the SENSE g-factors, even though the reconstruction will be performed by GRAPPA. The reason for this discrepancy is computational time: for SENSE g-factors, only the overlapping voxels should be taken into account, i.e. for an 8-fold acceleration the inversion of an 8x8 matrix is performed for each aliasing voxel group in each iteration. To compute GRAPPA g-factors, however, the reconstruction kernel needs to be calculated in each step, which means the inversion of a large matrix in each step that could make the iteration prohibitively long. However, the underlying mathematics of SENSE and GRAPPA are essentially similar, thus it is reasonable to expect a SENSE-optimal phase distribution to be close to the optimum for the given GRAPPA algorithm as well. Using GRAPPA instead of SENSE in the reconstruction also has a practical reason: for VCC-SENSE, not only the sensitivity profiles, but the actual object phase map is required, meaning that a second prescan would be necessary using the tailored pulses. However, VCC-GRAPPA does not require explicit phase information, and is therefore easier to use in most cases.

One limitation of the proposed technique is that the applied tailored excitation pulses are not sliceselective in either setup, as gradient waveforms were fixed to spirals to maintain feasible optimization time. Slice selection is performed only with the refocusing pulses, restricting the method to spin-echobased sequences when used for 2D imaging, and precluding interleaved multi-slice acquisition, as the whole volume is excited with every tailored pulse. However, incorporating slice selection into the excitation is theoretically possible with spokes pulses. To examine this possibility, simulations using slice-selective spokes pulses were performed, with the k-space location, amplitude, and phase of spokes simultaneously optimized, to introduce the target phase (see exemplary waveforms in Supporting Figure S6). Simulated performance of the resulting pulses (displayed in Supporting Figure S7) and reconstructed simulated datasets computed using the pulse profiles (shown in Supporting Figure S8) indicate that spokes pulses can achieve similar benefits as the spiral ones, while being slice-selective. Although the optimization of spokes pulses was prohibitively long for in-vivo measurements, their application could be feasible with more computational power, enabling interleaved multi-slice acquisitions. This could also allow the extension to gradient-echo-based sequences, in which phase variations are usually higher, and therefore the benefit of phase optimization is expected to be even greater. In these cases, the target phase distribution no longer resembles a linear ramp, and a notable reduction in g-factors can be achieved compared to a simple phase-ramp, as a gradient-echo-EPI example shows in Supporting Figure S2. Application of the method to 3D imaging is also entirely feasible using $3 \mathrm{D}$ pulses, although the current work was limited to $2 \mathrm{D}$ acquisitions. 
Another drawback is the long duration of the tailored pulses, and their consequently increased sensitivity to field inhomogeneity or system imperfections. Especially, this is the case with the SMS applications, where the pulse length increases with the number of slices with the used stack-of-spirals gradient waveform. However, pulse length could be significantly reduced by using parallel transmission, thus making higher slice accelerations feasible.
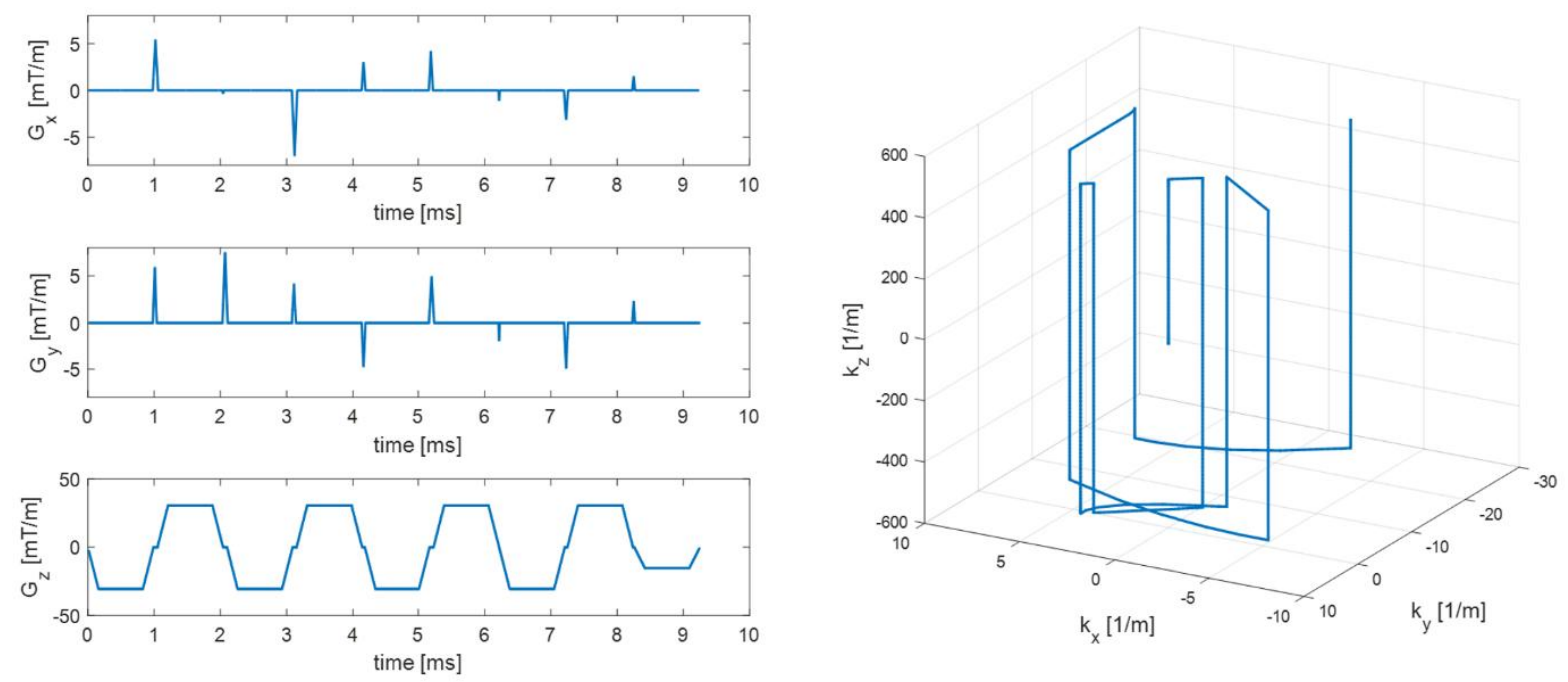

Supporting Figure S6. Exemplary gradient waveforms (left) and excitation k-space trajectory (right) for the spokes pulses designed for slice-selective tailored excitation. The designed RF pulses consist of sliceselective (in-plane case) or multi-slice-selective (SMS case) subpulses during the slice-direction gradient lobes. Amplitude and phase of the subpulses, as well as the in-plane location of the spokes were numerically optimized to introduce the target phase map.

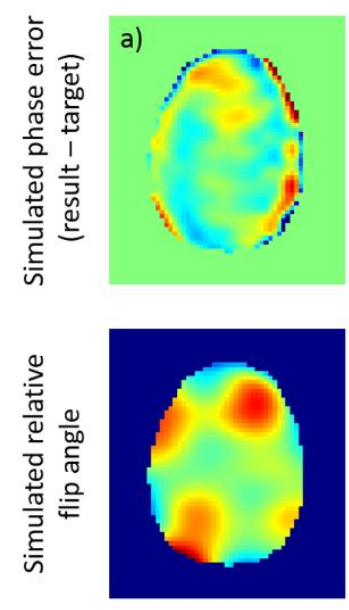

SE-EPI
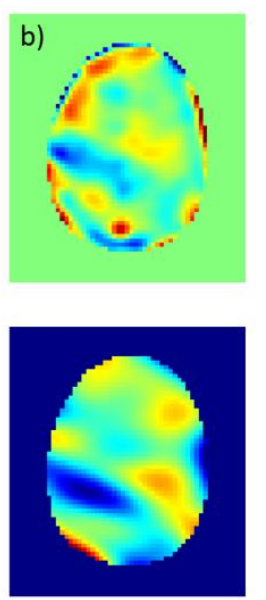

T1WSE
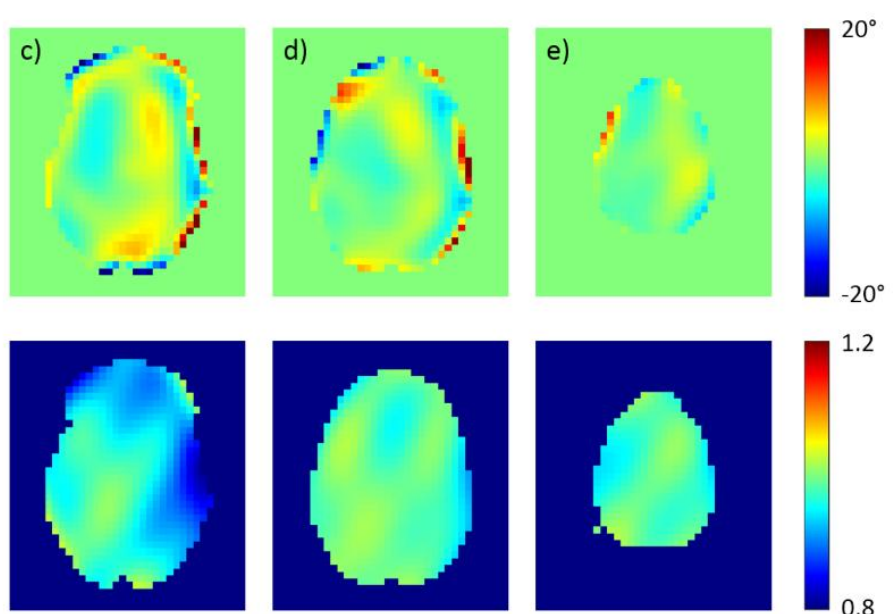

SMS EPI

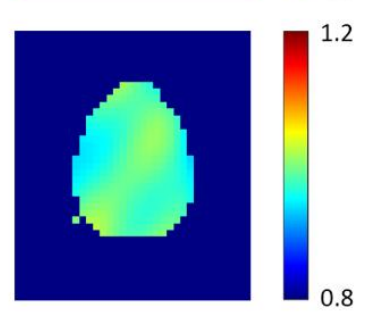

Supporting Figure S7. Simulated phase error (top row) and flip angle inhomogeneity (bottom row) of the designed spokes pulses, computed by numerical Bloch-simulation, for SE-EPI (a), T1-weighted spin-echo 
(b), and SMS EPI (c-e). Pulses were designed using the target phase maps calculated in the actual measurements. Possible system imperfections were disregarded in the simulation.
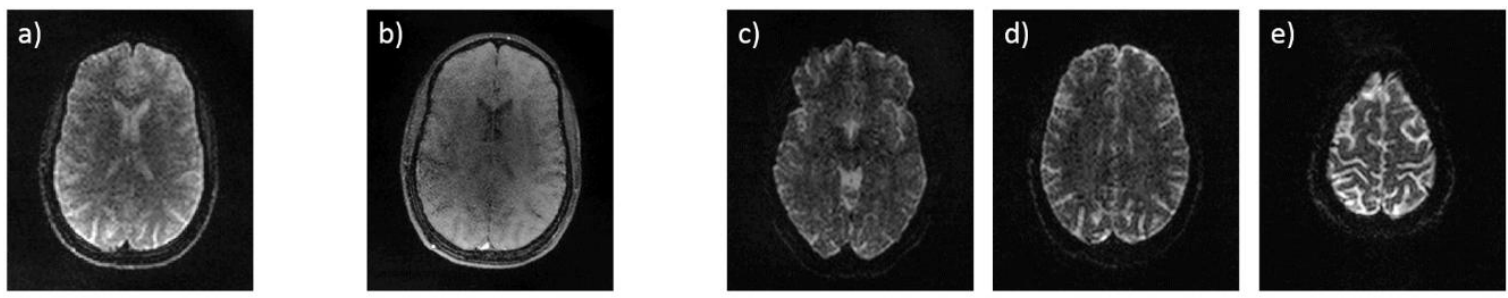

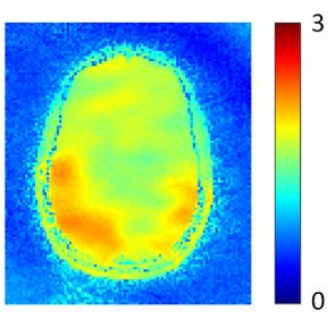

SE-EPI

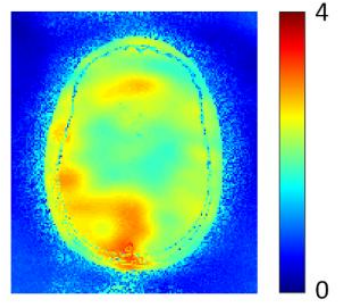

T1WSE
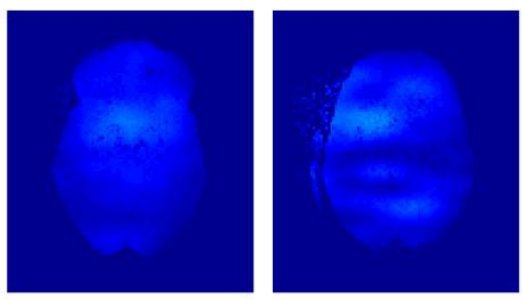

SMS EPI

Supporting Figure S8. Sum-of-squares VCC reconstructions (top row) and calculated g-factor maps (bottom row) of the datasets simulated by multiplying the conventional scans with the Bloch-simulated results of the spokes pulses. Results for SE-EPI (a), T1-weighted spin-echo (b), and SMS EPI (c-e) are displayed. g-Factor maps are scaled identically as the respective results shown in Figures 2, 3, and 6, for easier comparison.

\section{Conclusions}

We have shown that using phase-constrained reconstruction and tailored RF pulses designed to optimize the background phase of the object, noise amplification can be decreased significantly in both in-plane parallel imaging and simultaneous multi-slice acquisitions, while spatial flip angle homogeneity remains reasonable. Although these RF pulses have longer duration than conventional excitations, and their length increases even further with the number of slices in the SMS case, they could be shortened using parallel transmission, making higher slice accelerations feasible.

\section{Appendix 1: impact of background phase on VCC-SENSE g-factors}

Using $N$ coils and acceleration factor $R$, denoting the sensitivity of the $j$-th coil in the $k$-th position with $C_{j, k}$, the SENSE matrix and g-factors, neglecting noise correlations (1): 


$$
\mathbf{C}=\left[\begin{array}{ccc}
C_{1,1} & \cdots & C_{1, R} \\
\vdots & \ddots & \vdots \\
C_{N, 1} & \cdots & C_{N, R}
\end{array}\right], \quad g_{k}=\sqrt{\left(\left(\mathbf{C}^{H} \mathbf{C}\right)^{-1}\right)_{k, k}\left(\mathbf{C}^{H} \mathbf{C}\right)_{k, k}}
$$

Using column vectors, the elements of $\mathbf{C}^{H} \mathbf{C}$ are:

$$
\mathbf{c}_{k}=\left[\begin{array}{c}
C_{1, k} \\
C_{2, k} \\
\vdots \\
C_{N, k}
\end{array}\right], \quad\left(\mathbf{C}^{H} \mathbf{C}\right)_{j, k}=\mathbf{c}_{j}^{H} \mathbf{c}_{k}
$$

Calculating effective sensitivities by including the object phase at each position:

$$
\mathbf{D}=\left[\begin{array}{ccc}
C_{1,1} e^{i \varphi_{1}} & \cdots & C_{1, R} e^{i \varphi_{R}} \\
\vdots & \ddots & \vdots \\
C_{N, 1} e^{i \varphi_{1}} & \cdots & C_{N, R} e^{i \varphi_{R}}
\end{array}\right], \quad\left(\mathbf{D}^{H} \mathbf{D}\right)_{j, k}=\mathbf{c}_{j}^{H} \mathbf{c}_{k} \cdot e^{i\left(\varphi_{k}-\varphi_{j}\right)}
$$

Extending D with its conjugate:

$$
\begin{gathered}
\mathbf{D}_{\mathbf{V C C}}=\left[\begin{array}{c}
\mathbf{D} \\
\mathbf{D}^{*}
\end{array}\right] \\
g_{k}=\sqrt{\left(\left(\mathbf{D}_{\mathbf{V C C}}^{H} \mathbf{D}_{\mathbf{V C C}}\right)^{-1}\right)_{k, k}\left(\mathbf{D}_{\mathbf{V C C}}^{H} \mathbf{D}_{\mathbf{V C C}}\right)_{k, k}} \\
\mathbf{D}_{\mathbf{V C C}}^{H} \mathbf{D}_{\mathbf{V C C}}=\left[\begin{array}{c}
\mathbf{D} \\
\mathbf{D}^{*}
\end{array}\right]^{H}\left[\begin{array}{c}
\mathbf{D} \\
\mathbf{D}^{*}
\end{array}\right]=\mathbf{D}^{H} \mathbf{D}+\left(\mathbf{D}^{H} \mathbf{D}\right)^{*}=2 \cdot \operatorname{Re}\left(\mathbf{D}^{H} \mathbf{D}\right) \\
\left(\mathbf{D}_{\mathbf{V C C}}^{H} \mathbf{D}_{\mathbf{V C C}}\right)_{j, k}=2 \cdot \operatorname{Re}\left(\mathbf{c}_{j}^{H} \mathbf{c}_{k} \cdot e^{i\left(\varphi_{k}-\varphi_{j}\right)}\right)
\end{gathered}
$$

\section{Appendix 2: Cancellation of $B_{1}$ phase inhomogeneity in the tailored excitation}

The intrinsic object phase is introduced by $\mathrm{B}_{1}$ and $\mathrm{B}_{0}$ inhomogeneity. Supposing these two are independent:

$$
\varphi_{\text {intrinsic }}=\varphi_{\mathrm{B} 1}+\varphi_{\mathrm{B} 0}
$$

Target phase is the difference between optimal and intrinsic phases, neglecting $\mathrm{B}_{1}$ inhomogeneity:

$$
\varphi_{\text {target }}=\varphi_{\text {optimal }}-\varphi_{\text {intrinsic }}=\varphi_{\text {optimal }}-\left(\varphi_{\mathrm{B} 1}+\varphi_{\mathrm{B} 0}\right)
$$

Phase of the optimized scan is again affected by $\mathrm{B}_{1}$ and $\mathrm{B}_{0}$ inhomogeneity:

$$
\varphi_{\text {optimized }}=\varphi_{\text {target }}+\varphi_{\mathrm{B} 1}+\varphi_{\mathrm{B} 0}=\varphi_{\text {optimal }}-\left(\varphi_{\mathrm{B} 1}+\varphi_{\mathrm{B} 0}\right)+\varphi_{\mathrm{B} 1}+\varphi_{\mathrm{B} 0}=\varphi_{\text {optimal }}
$$


Therefore, if $\mathrm{B}_{1}$ and $\mathrm{B}_{0}$ inhomogeneities do not interact (e.g. during the pulse), the phase of the optimized measurement is not affected by $\mathrm{B}_{1}$ phase.

\section{Acknowledgements}

ÁK and ZV were supported by the Hungarian Brain Research Program (KTIA_13_NAP-AI/18). The authors thank James G. Pipe for spiral gradient waveform calculation code, and Brian A. Hargreaves for the numerical Bloch equation simulator.

\section{References}

1. Pruessmann KP, Weiger M, Scheidegger MB, Boesiger P. SENSE: sensitivity encoding for fast MRI. Magn Reson Med. 1999;42(5):952-962. [PubMed: 10542355]

2. Griswold MA, Jakob PM, Heidemann RM, Nittka M, Jellus V, Wang J, Kiefer B, Haase A. Generalized autocalibrating partially parallel acquisitions (GRAPPA). Magn Reson Med. 2002;47(6):1202-1210. [PubMed: 12111967]

3. Breuer FA, Kannengiesser SAR, Blaimer M, Seiberlich N, Jakob PM, Griswold MA. General formulation for quantitative G-factor calculation in GRAPPA reconstructions. Magn Reson Med. 2009;62(3):739-746. [PubMed: 19585608]

4. Larkman DJ, Hajnal JV, Herlihy AH, Coutts GA, Young IR, Ehnholm G. Use of multicoil arrays for separation of signal from multiple slices simultaneously excited. J Magn Reson Imaging. 2001;13(2):313-317. [PubMed: 11169840]

5. Barth M, Breuer F, Koopmans PJ, Norris DG, Poser BA. Simultaneous multi-slice (SMS) imaging techniques. Magn Reson Med. 2016;75(1):63-81. [PubMed: 26308571]

6. Setsompop K, Gagoski BA, Polimeni JR, Witzel T, Wedeen VJ, Wald LL. Blipped-controlled aliasing in parallel imaging for simultaneous multi-slice echo planar imaging with reduced g-factor penalty. Magn Reson Med. 2012;67(5):1210-1224. [PubMed: 21858868]

7. Feinberg DA, Moeller S, Smith SM, Auerbach E, Ramanna S, Gunther M, Glasser MF, Miller KL, Ugurbil K, Yacoub E. Multiplexed echo planar imaging for sub-second whole brain FMRI and fast diffusion imaging. PloS One. 2010;5(12):e15710. [PubMed: 21187930]

8. Willig-Onwuachi JD, Yeh EN, Grant AK, Ohliger MA, McKenzie CA, Sodickson DK. Phaseconstrained parallel MR image reconstruction. J Magn Reson. 2005;176(2):187-98. [PubMed: 16027017] 
9. Samsonov AA, Kholmovski EG, Parker DL, Johnson CR. POCSENSE: POCS-based reconstruction for sensitivity encoded magnetic resonance imaging. Magn Reson Med. 2004;52(6):1397-406. [PubMed: $15562485]$

10. Blaimer M, Gutberlet M, Kellman P, Breuer FA, Köstler H, Griswold MA. Virtual coil concept for improved parallel MRI employing conjugate symmetric signals. Magn Reson Med. 2009;61(1):93-102. [PubMed: 19097211]

11. Lew C, Pineda AR, Clayton D, Spielman D, Chan F, Bammer R. SENSE phase-constrained magnitude reconstruction with iterative phase refinement. Magn Reson Med. 2007;58(5):910-921. [PubMed: 17969127]

12. Noll DC, Nishimura DG, Macovski A. Homodyne detection in magnetic resonance imaging. IEEE Trans Med Imaging 1991;10:154-163. [PubMed: 18222812]

13. McGibney G, Smith MR, Nichols ST, Crawley A. Quantitative evaluation of several partial fourier reconstruction algorithms used in mri. Magn Reson Med. 1993;30(1):51-59. [PubMed: 8371675]

14. Bydder M, Robson MD. Partial fourier partially parallel imaging. Magn Reson Med. 2005;53(6):1393-1401. [PubMed: 15906299]

15. Blaimer M, Heim M, Neumann D, Jakob PM, Kannengiesser S, Breuer FA. Comparison of phaseconstrained parallel MRI approaches: Analogies and differences. Magn Reson Med. 2016;75(3):10861099. [PubMed: 25845973]

16. Blaimer M, Choli M, Jakob PM, Griswold MA, Breuer FA. Multiband phase-constrained parallel MRI. Magn Reson Med. 2013;69(4):974-980. [PubMed: 23440994]

17. Schneider JT, Blaimer M, Ullmann P. Tailoring the image background phase by spatially selective excitation for improved parallel imaging reconstruction performance. Proceedings of the 20th Annual Meeting of ISMRM, Melbourne, Australia; 2012. p. 516

18. Blaimer M, Breuer FA, Seiberlich N, Mueller MF, Heidemann RM, Jellus V, Wiggins G, Wald LL, Griswold MA, Jakob PM. Accelerated volumetric MRI with a SENSE/GRAPPA combination. J Magn Reson Imaging. 2006;24(2):444-450. [PubMed: 16786571]

19. Lustig M, Pauly JM. SPIRiT: Iterative self-consistent parallel imaging reconstruction from arbitrary k-space. Magn Reson Med. 2010;64(2):457-471. [PubMed: 20665790]

20. Uecker M, Lai P, Murphy MJ, Virtue P, Elad M, Pauly JM, Vasanawala SS, Lustig M. ESPIRiT_an eigenvalue approach to autocalibrating parallel MRI: Where SENSE meets GRAPPA. Magn Reson Med. 2014;71(3):990-1001. [PubMed: 23649942] 
21. Kellman P, McVeigh ER. Image Reconstruction in SNR Units: A General Method for SNR Measurement. Magn Reson Med. 2005;54(6):1439-1447. [PubMed: 16261576]

22. Wang Z, Wang J, Detre JA. Improved data reconstruction method for GRAPPA. Magn Reson Med. 2005;54(3):738-742. [PubMed: 16088880]

23. Breuer FA, Blaimer M, Heidemann RM, Mueller MF, Griswold MA, Jakob PM. Controlled aliasing in parallel imaging results in higher acceleration (CAIPIRINHA) for multi-slice imaging. Magn Reson Med. 2005;53(3):684-691. [PubMed: 15723404]

24. Breuer FA, Blaimer M, Mueller MF, Seiberlich N, Heidemann RM, Griswold MA, Jakob PM. Controlled aliasing in volumetric parallel imaging (2D CAIPIRINHA). Magn Reson Med. 2006;55(3):549-556. [PubMed: 16408271]

25. Lagarias J, Reeds J, Wright M, Wright P. Convergence Properties of the Nelder--Mead Simplex Method in Low Dimensions. SIAM Journal on Optimization. 1998;9(1):112-147.

26. MRI UNBOUND website, Spiral Waveform Generation code by James G. Pipe, 2012. Available at http://www.ismrm.org/mri unbound/sequence.htm. Accessed March 10, 2016.

27. Yip C, Fessler JA, Noll DC. Iterative RF pulse design for multidimensional, small-tip-angle selective excitation. Magn Reson Med. 2005;54(4):908-917. [PubMed: 16155881]

28. Zhao T, Hu X. Iterative GRAPPA (iGRAPPA) for improved parallel imaging reconstruction. Magn Reson Med. 2008;59(4):903-907. [PubMed: 18383282]

29. Hargreaves BA. Bloch equation simulator, 2003. Available at http://mrsrl.stanford.edu/ brian/blochsim/. Accessed March 9, 2016.

30. Kettinger A, Kannengiesser SAR, Breuer FA, Vidnyanszky Z, Blaimer M. Tailored RF pulses for optimized phase-constrained parallel imaging: initial experience on a clinical system. Proceedings of the 33rd Annual Scientific Meeting of ESMRMB, Vienna, Austria; 2016. p. 380

31. Kettinger A, Kannengiesser SAR, Breuer FA, Vidnyanszky Z, Blaimer M. Tailored 3D RF pulses for g-factor reduction in phase-constrained simultaneous multi-slice imaging. Proceedings of the 25th Annual Meeting of ISMRM, Hawaii, USA; 2017. p. 3834 